\title{
On self-sustained oscillations in two-dimensional compressible flow over rectangular cavities
}

\author{
By CLARENCE W. ROWLEY†, TIM COLONIUS \\ AND AMIT J. BASU† \\ California Institute of Technology, Pasadena, CA 91125, USA
}

(Received 18 November 1999 and in revised form 24 September 2001)

Numerical simulations are used to investigate the resonant instabilities in twodimensional flow past an open cavity. The compressible Navier-Stokes equations are solved directly (no turbulence model) for cavities with laminar boundary layers upstream. The computational domain is large enough to directly resolve a portion of the radiated acoustic field, which is shown to be in good visual agreement with schlieren photographs from experiments at several different Mach numbers. The results show a transition from a shear-layer mode, primarily for shorter cavities and lower Mach numbers, to a wake mode for longer cavities and higher Mach numbers. The shear-layer mode is characterized well by the acoustic feedback process described by Rossiter (1964), and disturbances in the shear layer compare well with predictions based on linear stability analysis of the Kelvin-Helmholtz mode. The wake mode is characterized instead by a large-scale vortex shedding with Strouhal number independent of Mach number. The wake mode oscillation is similar in many ways to that reported by Gharib \& Roshko (1987) for incompressible flow with a laminar upstream boundary layer. Transition to wake mode occurs as the length and/or depth of the cavity becomes large compared to the upstream boundary-layer thickness, or as the Mach and/or Reynolds numbers are raised. Under these conditions, it is shown that the Kelvin-Helmholtz instability grows to sufficient strength that a strong recirculating flow is induced in the cavity. The resulting mean flow is similar to wake profiles that are absolutely unstable, and absolute instability may provide an explanation of the hydrodynamic feedback mechanism that leads to wake mode. Predictive criteria for the onset of shear-layer oscillations (from steady flow) and for the transition to wake mode are developed based on linear theory for amplification rates in the shear layer, and a simple model for the acoustic efficiency of edge scattering.

\section{Introduction}

Oscillations in the flow past an open cavity have been studied for decades, but still there remain many open questions about even the basic physical mechanisms underlying the self-sustained oscillations. Cavity oscillations in compressible flows are typically described as a flow-acoustic resonance phenomenon, and its first detailed description is credited to Rossiter (1964), but it was known earlier as a mechanism for edge tones (e.g. Powell 1953, 1961). In this mechanism, small disturbances in the free shear layer spanning the cavity are amplified via Kelvin-Helmholtz instability. Their interaction with the trailing cavity edge gives rise to an unsteady, irrotational field,

$\dagger$ Present address: Princeton University, Princeton, NJ 08544, USA. $\ddagger$ Present address: Bridge Information Systems, Inc., Palo Alto, CA 94303, USA. 
the upstream influence of which excites further disturbances to the free shear layer, especially near the cavity leading edge. At very low Mach number, the scattered field is essentially incompressible, and the feedback to the leading edge is instantaneous, while at moderate Mach number there is an acoustic delay. Resonance occurs at frequencies where the phase change of the disturbance leads to constructive reinforcement. In this paper we consider Mach numbers from 0.2 to 0.8 , and simply refer to the feedback field as 'acoustic'.

Another mode of cavity oscillation has been observed, but has received much less attention, and is relatively poorly understood. In incompressible experiments for an axisymmetric cavity, Gharib \& Roshko (1987) observed a wake mode, where the oscillating flow over the cavity resembles the wake behind a bluff body, rather than a free shear layer. Flow features in this wake mode were qualitatively very different from those in the shear-layer mode described by Rossiter, and wake mode was accompanied by a large increase in drag. Similar dramatic increases in drag had been previously observed by Fox (1968) as the cavity length was increased, in flows with thin laminar upstream boundary layers, and Roshko (1955) observed an intermittency 'analogous to the large fluctuations of drag which occur on a bluff cylinder in the critical range of Reynolds number,' where the flow may be switching between shear-layer mode and a type of wake mode. Recent experiments and numerical studies by Kriesels et al. (1995) for a flow past closed branches of a pipe (with both circular and two-dimensional cross-sections) also demonstrate flow structures that closely resemble wake mode.

Note that cavity oscillations have been categorized by Rockwell \& Naudascher (1978) into 'fluid-dynamic' oscillations, and 'fluid-resonant' oscillations, where the acoustic resonance of the cavity itself plays an important role, as in a flute or an organ pipe. Both of these classes of oscillations are variations of shear-layer mode, and should not be confused with wake mode.

Research in cavity flows has seen renewed activity in recent years, largely because of the possibility of using active control to reduce the oscillations, for applications such as aircraft wheel wells and weapons bays. For active control to be used most effectively, an accurate understanding of the flow physics is essential. This was the original motivation for the present study. The recent literature on control of cavity oscillations is too vast to survey here, but we mention the early work of Sarohia \& Massier (1977) and Gharib (1987), and refer to the recent review article by Colonius (2001) for more information. More details about cavity oscillations in general may be found in review articles (e.g. Rockwell \& Naudascher 1978; Blake \& Powell 1986); see also the recent work by Howe (1997) for very low Mach number cavity flows, and Crighton (1992) for edge tones.

The focus of the present work is to investigate numerically the two regimes mentioned above-shear-layer mode and wake mode-in two-dimensional subsonic flow over a rectangular cavity. The experiments of Gharib \& Roshko were performed for an axisymmetric cavity, and when wake mode is present they mention significant large-scale motion across the centreline, suggesting that wake mode oscillations might be suppressed in two-dimensional geometries. The experiments of Kriesels et al. (1995) also involved coupling between two different cavities (closed side branches of a pipe), and it is not clear to what extent the coupling is important. The present investigation demonstrates that wake mode occurs in two-dimensional flows even without the presence of such coupling.

Previous numerical studies of compressible cavity flows have used the twodimensional unsteady RANS (Reynolds averaged Navier-Stokes) equations with a $k-\epsilon$ turbulence model (Lamp \& Chokani 1997; Zhang, Rona \& Edwards 1998; 
Fuglsange \& Cain 1992). The effectiveness of compressible turbulence models for separated oscillating flows, and especially their radiated acoustic field (which, as noted above, is an integral part of the resonant instability modes) remains an open question. Direct numerical simulations (DNS) provide a means to study the details of the modes of oscillation and their interactions, albeit at low Reynolds number, and have been used recently by Colonius, Basu \& Rowley (1999) and Shieh \& Morris (1999). In $\S 2$ we present a high-order-accurate method for solving the compressible Navier-Stokes equations in the cavity geometry and give results for two-dimensional computations. The generated acoustic field is directly resolved in the computation, and the domain is made large enough to include a portion of the radiated acoustic field. It needs to be stressed that in the context of two-dimensional flows, we use the term 'direct' simulation to imply that there is no turbulence model. In this case the flow is an unstable laminar flow that is confined to evolve in only two dimensions. The turbulent cavity flow is of course three-dimensional, but it is thought that in many cases the resonant modes are approximately two-dimensional. Some two-dimensional Navier-Stokes calculations of the cavity flow were also undertaken by Slimon, Davis \& Wagner (1998), who formulated the problem as a 'hybrid' method where, for low Mach number, the acoustic modes were solved independently of the hydrodynamic field.

In $\S 3$, results from the computation are used to visualize the acoustic feedback process described above, and the radiated acoustic fields are compared to the schlieren photographs of Krishnamurty (1956). Details of the shear layer spanning the cavity are discussed, and compared with available experimental results and predictions from linear theory.

In $\S 4$, we consider wake mode oscillations in detail, and find that the frequencies of oscillation are independent of Mach number, suggesting that acoustics no longer play a role in the mechanism for self-sustained oscillations. We conjecture that for sufficiently strong recirculating flows within the cavity, the shear layer becomes absolutely unstable (in the sense of Huerre \& Monkewitz 1985), and thus provides a competing feedback mechanism, separate from the usual acoustic feedback. The transition to wake mode appears also to be connected with unsteady oscillating separation of the boundary layer upstream of the cavity.

In $\S 5$, we develop criteria to predict both the onset of fluctuations in shear-layer mode (from steady flow), and the transition to wake mode. The criteria are based on linear stability calculations of the amplification of disturbances in the shear layer, and a simple model for the efficiency of radiation from the cavity trailing edge. The criteria are in excellent agreement with the computed results. The model also sheds some light on the parametric dependence of the onset of fluctuations, explaining trends seen in previous experiments, and also the transition to wake mode. Finally, in $\S 6$, we discuss the wake mode transition in the context of experimental results and some previous RANS calculations, and propose explanations for why wake mode is less likely to appear in three-dimensional flows and at higher Reynolds numbers.

\section{Numerical method}

The numerical method used here is very similar to methods used previously for direct computations of sound generation in mixing layers and jets and other canonical problems (e.g. Colonius, Lele \& Moin 1997), wherein the fully compressible NavierStokes equations are solved. These studies have shown the efficacy of sixth-orderaccurate compact finite-difference schemes (Lele 1992) in resolving acoustic fields 


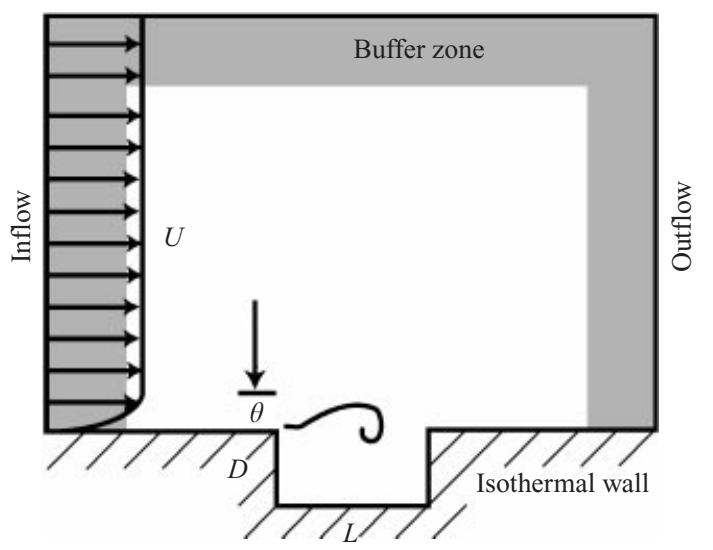

FIGURE 1. Schematic diagram of cavity configuration and computational domain.

with velocity fluctuations five orders of magnitude smaller than near-field fluctuations (Colonius et al. 1997). Time integration is performed with a fourth-order Runge-Kutta method. This combination of schemes results in very low numerical dissipation, which allows accurate wave propagation. The method relies solely on physical viscosity for stability.

Boundary conditions play a key role in aeroacoustic computations. Artificial boundaries (inflow/outflow/normal) must allow vortical and acoustic waves to pass freely with minimal reflection. It is important to distinguish between two types of reflections that may occur: 'smooth' reflections, which arise due to approximations in the continuous boundary condition formulation; and 'spurious' or saw-tooth reflections, which arise due to the dispersive nature of finite difference schemes (Rowley \& Colonius 2000). For equations of motion linearized about a uniform mean flow, it is possible to derive boundary conditions that are non-reflecting for both types of waves, to arbitrarily high order of accuracy (Rowley \& Colonius 2000). For nonlinear equations, especially at outflow boundaries, the interaction of disturbance amplitudes and mean flow gradients severely limits the accuracy of any linear boundary conditions (Colonius, Lele \& Moin 1993). Several treatments that rely on a 'buffer' zone near the computational boundary have been suggested to remedy this situation. These include combinations of grid stretching and filtering (e.g. Colonius et al. 1993), and the addition of artificial convection velocities and damping terms to the equations (e.g. Freund 1997). These 'buffer' conditions are combined with robust (but low-order accurate) non-reflecting boundary conditions, such as one-dimensional characteristic wave decompositions (Thompson 1987; Poinsot \& Lele 1992).

Figure 1 shows a schematic diagram of the computational domain. A Cartesian grid is used, with clustering of nodes near all the walls. Analytical error function mappings are used for the grid stretching. The code is parallelized using a domain decomposition method. Typical grids (see captions of figures 2 and 3) contain about half a million grid points. The code has been run on 8 to 32 processors of an IBM SP2. The wall is assumed to be isothermal at the same temperature as the free stream (therefore transport properties are assumed constant, and the Prandtl number is taken as 0.7 ). For the inflow, outflow and normal boundaries, the one-dimensional boundary conditions of Poinsot \& Lele (1992) are used, together with artificial damping terms in a buffer region (Freund 1997). These terms, of the form $\sigma\left(\boldsymbol{q}-\boldsymbol{q}_{\text {target }}\right)$ are added to the right-hand sides of the equations in conservative form (here $q$ is a vector of the 

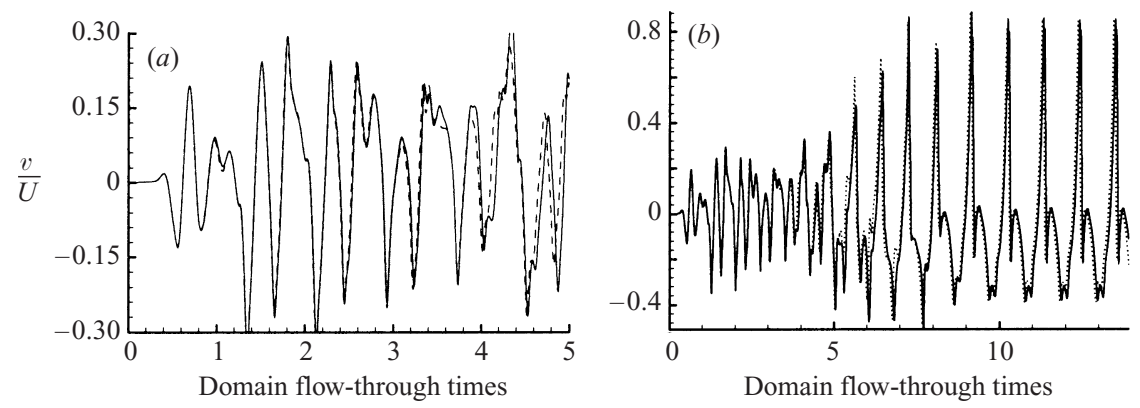

FIGURE 2. Effect of boundary position and grid resolution on the normal velocity at $y=0$ and $x=3.13 D:(a)$ reference case L4 (- compared with a finer grid case (---) and larger domain case $(\cdots \cdots) ;(b)$ only the larger domain case, for longer time. Reference case L4 has downstream boundary $10.6 D$, upstream $-4.3 D$, normal $9.2 D$. The grid has $1152 \times 384$ points above the cavity in the streamwise and spanwise directions, respectively, and $384 \times 94$ points in the cavity. The finer grid case has the same boundaries as run L4, but $50 \%$ more grid points (in each direction). The larger domain case extends to $15 \mathrm{D}$ downstream and $15 \mathrm{D}$ in the normal direction. Note that the dotted line falls nearly directly on top of the solid line in $(a)$.

conservative dependent variables). The damping, $\sigma$, varies smoothly from a constant value at the boundary to zero at the edge of the buffer (i.e. the edge of the 'physical' portion of the computational domain). For the isothermal wall boundary conditions, including the cavity edges, the formulation recommended by Poinsot \& Lele is used.

The simulations are initiated by spanning the cavity with a Blasius flat-plate boundary layer. The following parameters may be independently varied: the length of the cavity relative to the initial boundary-layer thickness at the cavity leading edge, $L / \theta_{0}$; the Reynolds number, $R e_{\theta}=\rho_{\infty} U \theta_{0} / \mu$, where $\rho_{\infty}$ is ambient density, $U$ is free-stream velocity and $\mu$ viscosity; the Mach number of the free stream, $M=U / a_{\infty}$ where $a_{\infty}$ is ambient sound speed; and the cavity length to depth ratio, $L / D$. Because of the expense, only a relatively small portion of parameter space may be investigated. In the present paper, we concentrate on two-dimensional computations with laminar upstream boundary layers. Table 1 shows relevant parameters for the runs performed.

It should be noted that the quoted values of $\theta_{0}$, the boundary-layer momentum thickness at the upstream cavity edge, are taken from the initial condition for each run. The oscillations that develop in the cavity can alter this value. The variation is very slight in the case of the shear-layer mode oscillations, but can be significant in the wake mode discussed in $\S 4$.

\subsection{Validation}

For the present flow, and for resonant flows in general, it is of critical importance that results be independent of the location of the boundaries, and the boundary treatment. If boundaries are not treated properly, repeated spurious reflections of waves can lead to self-forcing of the flow, in a process that is ultimately indistinguishable from physical instability (e.g. Colonius et al. 1993). We have run several cases with variable boundary location and grid spacing, in order to find appropriate boundary locations, as well as demonstrating grid convergence. The results of two such tests are shown in figures 2 and 3.

Figure 2 shows the normal velocity at a single point in the shear layer $(x=3.13 D$, $y=0$ ), for different grid resolutions and boundary locations, but otherwise using the same parameters as run L4. Other probe locations yielded similar results. Note that time is normalized by the free-stream velocity and the total length of the 


\begin{tabular}{|c|c|c|c|c|c|c|c|}
\hline Run & $L / D$ & $L / \theta_{0}$ & $M$ & $R e_{\theta}$ & Mode & $S t_{D}$ (wake mode) & $C_{D}$ \\
\hline L1 & 1 & 20.3 & 0.6 & 73.9 & $\mathrm{NO}$ & - & 0.002 \\
\hline L2 & 2 & 52.8 & 0.6 & 56.8 & SL & - & 0.008 \\
\hline L3 & 3 & 75.0 & 0.6 & 60.0 & $\mathrm{M}$ & - & 0.031 \\
\hline L4 & 4 & 102.1 & 0.6 & 58.8 & $\mathrm{~W}$ & 0.064 & 0.227 \\
\hline L5 & 5 & 123.3 & 0.6 & 60.8 & W & 0.054 & 0.404 \\
\hline $4 \mathrm{M} 2$ & 4 & 102 & 0.2 & 58.8 & SL & - & \\
\hline $4 \mathrm{M} 3$ & 4 & 102 & 0.3 & 58.8 & $\mathrm{M}$ & - & \\
\hline $4 \mathrm{M} 4$ & 4 & 102 & 0.4 & 58.8 & W & 0.064 & \\
\hline 4M5 & 4 & 102 & 0.5 & 58.8 & W & 0.064 & \\
\hline 4M6 (L4) & 4 & 102 & 0.6 & 58.8 & W & 0.064 & \\
\hline $4 \mathrm{M} 7$ & 4 & 102 & 0.7 & 58.8 & W & 0.061 & \\
\hline $4 \mathrm{M} 8$ & 4 & 102 & 0.8 & 58.8 & W & 0.061 & \\
\hline $2 \mathrm{M} 2$ & 2 & 52.8 & 0.2 & 56.8 & NO & 一 & \\
\hline $2 \mathrm{M} 3$ & 2 & 52.8 & 0.3 & 56.8 & $\mathrm{NO}$ & - & \\
\hline $2 \mathrm{M} 4$ & 2 & 52.8 & 0.4 & 56.8 & SL & - & \\
\hline $2 \mathrm{M} 5$ & 2 & 52.8 & 0.5 & 56.8 & SL & - & \\
\hline 2M6 (L2) & 2 & 52.8 & 0.6 & 56.8 & SL & - & \\
\hline $2 \mathrm{M} 7$ & 2 & 52.8 & 0.7 & 56.8 & SL & - & \\
\hline $2 \mathrm{M} 8$ & 2 & 52.8 & 0.8 & 56.8 & SL & - & \\
\hline $\mathrm{H} 1$ & 1 & 23.2 & 0.6 & 86.3 & NO & - & \\
\hline $\mathrm{H} 2$ & 2 & 58.4 & 0.6 & 68.5 & SL & - & \\
\hline H3 & 3 & 84.9 & 0.6 & 70.6 & M & - & \\
\hline H4 & 4 & 116.7 & 0.6 & 68.5 & W & 0.063 & \\
\hline LG6a & 6 & 45.18 & 0.6 & 58.57 & $\mathrm{NO}$ & - & \\
\hline LG6b & 6 & 90.36 & 0.6 & 29.28 & SL & - & \\
\hline LG8 & 8 & 60.24 & 0.6 & 58.57 & $\mathrm{NO}$ & - & \\
\hline TK4a & 4 & 30.12 & 0.6 & 58.6 & $\mathrm{NO}$ & - & \\
\hline TK4b & 4 & 60.24 & 0.6 & 58.8 & SL & - & \\
\hline TK4c & 4 & 75.30 & 0.6 & 58.8 & M & - & \\
\hline TK4d (L4) & 4 & 102.1 & 0.6 & 58.8 & W & 0.064 & \\
\hline R4a & 4 & 86.06 & 0.6 & 45.8 & SL & - & \\
\hline R4b (TK4b) & 4 & 60.24 & 0.6 & 58.8 & SL & - & \\
\hline $\mathrm{R} 4 \mathrm{c}$ & 4 & 74.55 & 0.6 & 80.5 & W & 0.063 & \\
\hline
\end{tabular}

TABle 1. Parameters for the different computer runs. Abbreviations for modes are: $\mathrm{NO}=$ no oscillations, $\mathrm{SL}=$ shear layer, $\mathrm{W}=$ wake, $\mathrm{M}=$ mixed. Strouhal numbers of vortex shedding (based on cavity depth and free-stream velocity) are given for wake mode runs, and the computed drag coefficient is given for run series L (see $\S 4$ ).

computational domain for the reference case. The larger-domain case is run for longer than the finer-grid case, as the domain size is more likely to cause lowfrequency errors, arising from non-physical reflections at boundaries. Figure 3 shows a similar test for run L2, and the spectra are compared.

For the first 3 to 4 flow-through times, the results are nearly identical, independent of grid resolution and boundary location. Small differences are apparent at later times, which is not unexpected given the sensitive dependence on initial conditions. For run L2, greater differences are observed after about 10 flow-through times, but the spectra are almost identical. We conclude that the boundary locations and grid resolutions for runs L2 and L4 are adequate, and similar locations and resolutions were used in the other runs in table 1. 

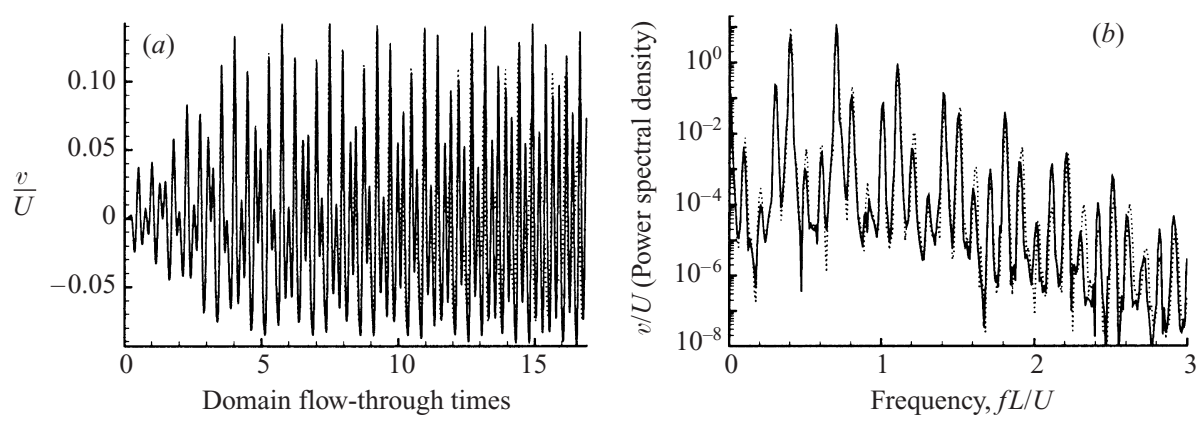

FIGURE 3. Effect of grid placement on the normal velocity at $y=0$ and $x=1.57 D$, for run L2: $(a)$ the time trace for the reference case $(-)$ and for a larger domain case $(\cdots \cdots) ;(b)$ the spectra of the data in $(a)$. Reference case L2 has downstream boundary 7.6D, upstream $-3.9 D$, normal $9.2 \mathrm{D}$. The grid has $1008 \times 384$ points above the cavity, and $240 \times 96$ points in the cavity. The larger domain case extends to $11.8 \mathrm{D}$ downstream and $15.6 \mathrm{D}$ in the normal direction.
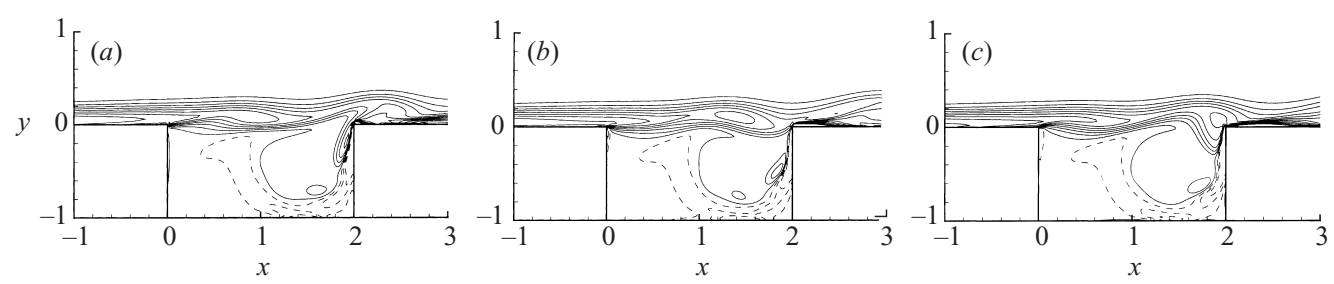

FIGURE 4. Instantaneous vorticity contours for run L2 (shear-layer mode) at three different times $(a-c)$, corresponding to approximately one-third phase intervals of the dominant mode of oscillation (2nd Rossiter mode). 15 equi-spaced contours between $\omega D / U=-5$ and 1.67 are shown; positive contours are dashed. Only a small portion of the computational domain near the cavity is shown.

\section{Shear-layer mode}

The shear-layer mode is characterized by the feedback process described in the introduction: the roll-up of vorticity in the shear layer, impingement and scattering of acoustic waves at the downstream cavity edge, upstream acoustic wave propagation, and receptivity of the shear layer to acoustic disturbances. The process is clearly born out by the computational results. Iso-contours of vorticity are depicted in figure 4 for run L2, and these are indicative of all the runs in the shear-layer mode of oscillation. Vortical disturbances in the shear layer are clearly evident, and the flow inside the cavity is relatively quiescent, with a weak vortex occupying the downstream half of the cavity. Vorticity of the opposite sign (to boundary-layer vorticity) is generated along the walls of the cavity. Note that at three different instants in time, while the phase of the disturbances in the shear layer has shifted, the vorticity contours in the cavity are nearly the same. The steadiness of the vortex occupying the latter half of the cavity (which is confirmed by the mean flow, discussed in comparison to the wake mode mean flow in $\S 4$ ) indicates that the interaction of the flow inside the cavity with the shear layer is relatively weak.

Figure 5 provides a qualititative confirmation of the acoustic feedback process. Plotted are contours of the density fluctuations along the cavity mouth $(y=0)$ and along the cavity walls, as a function of time. Both upstream (acoustic) and downstream (vortical) disturbances are evident along the shear layer, while density fluctuations along the bottom are purely acoustic. The phase variation of these disturbances will 

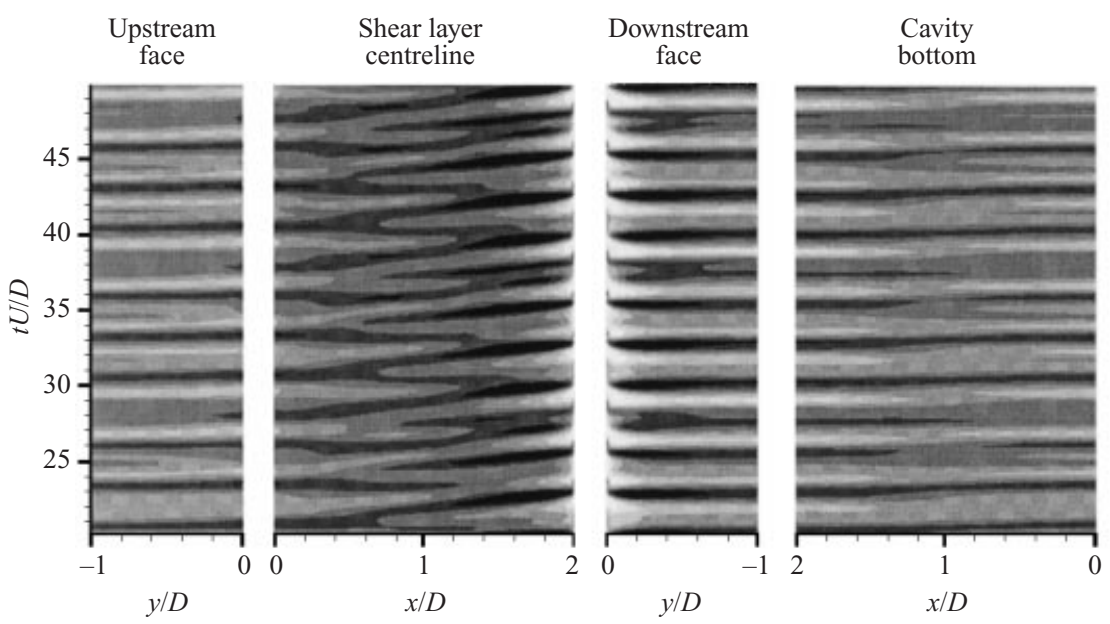

FiguRE 5. Diagram showing contours of density perturbation along the cavity edges and cavity mouth (shear layer), as a function of time, for run L2. Contour levels range from -0.03 (dark) to 0.03 (light).

be examined in more detail in $\S 3.4$, but figure 5 is a useful visualization of the feedback loop involved.

\subsection{Acoustic field}

In general, it is not possible to distinguish objectively between acoustic and hydrodynamic fluctuations in a complex, vortical flow. In this section, we examine the acoustic field produced by the cavity, where we loosely define the acoustic field as the irrotational field generated as vorticity is swept past the trailing corner of the cavity.

The density fluctuations produced by the self-sustained oscillations are shown in figure 6, where we compare the present results to schlieren photographs taken by Krishnamurty (1956) for Mach numbers $0.64,0.7$, and 0.8 , and $L / D=2$. The experimental conditions are similar, in that the incoming boundary layer is laminar, but the Reynolds number is higher by a factor of about 5 . The cavity in the experiment had a transverse length of 4 in., compared to the depth of $0.1 \mathrm{in}$., and it is expected under these conditions that the instabilities are approximately twodimensional. There is a very good qualitative agreement between experiment and computation. The greyscale contour levels in the simulation were chosen to provide the best visual comparison with the experiment. It can be noted from the plots that the frequencies of oscillation (and hence wavelength of the acoustic field) are quite close at $M=0.7$ and $M=0.8$. At $M=0.6$ ( 0.64 for the experiment), it appears that the frequency of oscillation is substantially lower in the simulation, and the experiment at these conditions is dominated by Rossiter mode 2 while the computation is dominated by Rossiter mode 1 (see $\S 3.2$ ).

Also note from figure 6 that the radiation is intense enough that there is significant steepening of the compressions as the waves propagate, especially at the higher Mach numbers.

Sound pressure levels (SPL) for the acoustic field above the cavity are shown in figure 7 for $M=0.6$. Maximum SPL near the downstream cavity edge is about $180 \mathrm{~dB}$, and peak radiation to the far field occurs at an angle of about $135^{\circ}$ from the 
(a) Schlieren, $M=0.64$

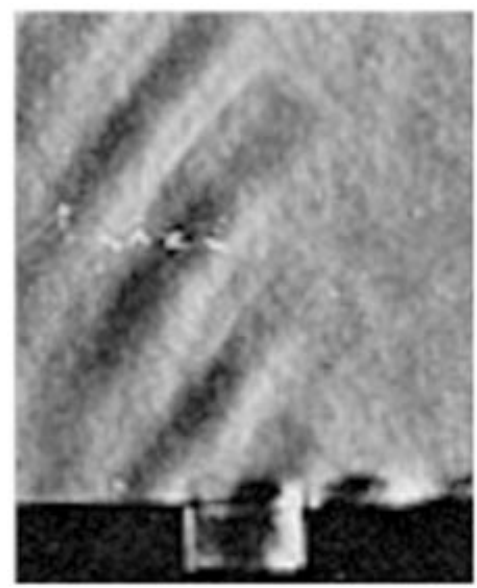

(c) Schlieren, $M=0.7$

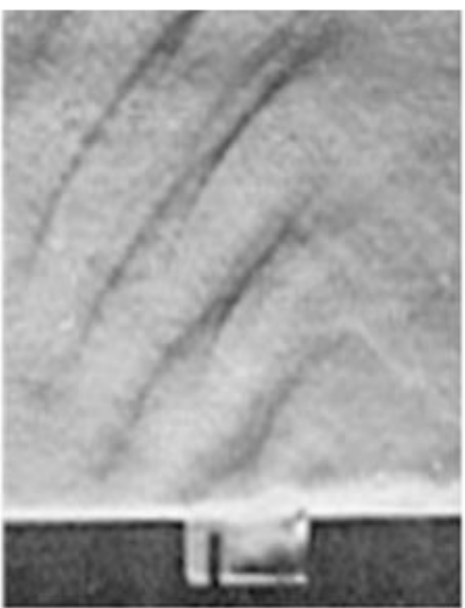

(e) Schlieren, $M=0.8$

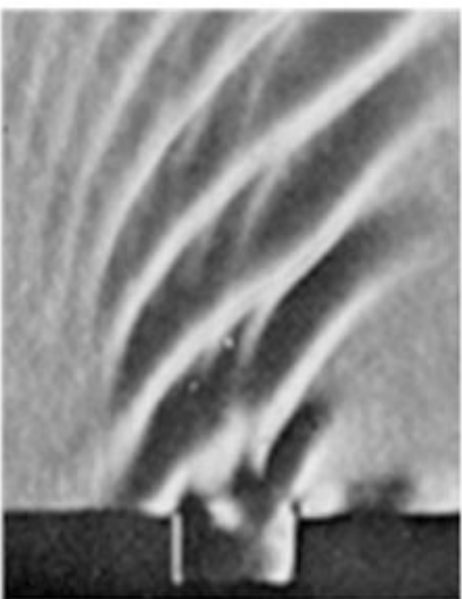

(b) Run 2M6, $M=0.6$

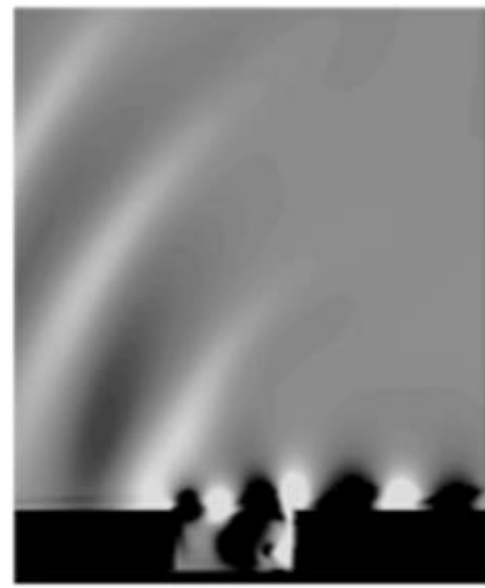

(d) Run $2 \mathrm{M} 7, M=0.7$

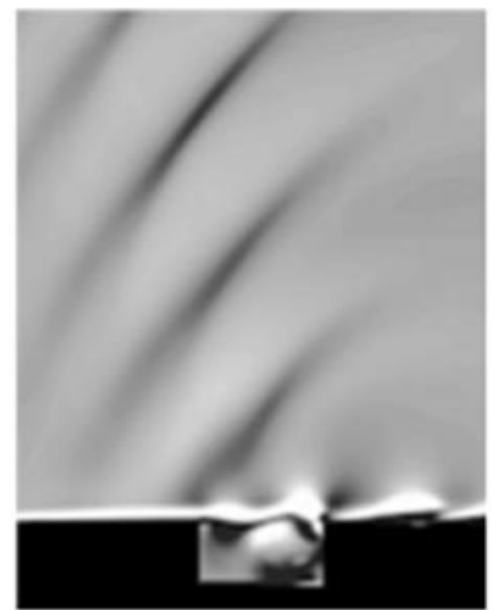

( $f$ ) Run $2 \mathrm{M} 8, M=0.8$

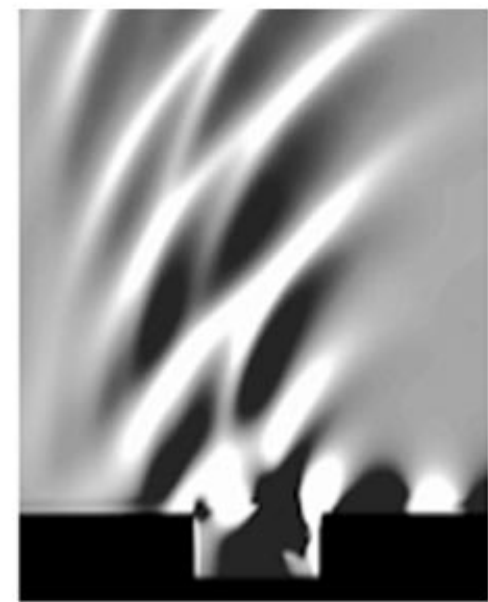

FIGURE 6. Comparison of schlieren photographs (Krishnamurty 1956) with contours of density gradient from the DNS. In the schlieren photographs, the knife edge is horizontal in $(c)$, vertical in the others; in the DNS figures, $\partial \rho / \partial y$ is shown in $(d), \partial \rho / \partial x$ in the others. 


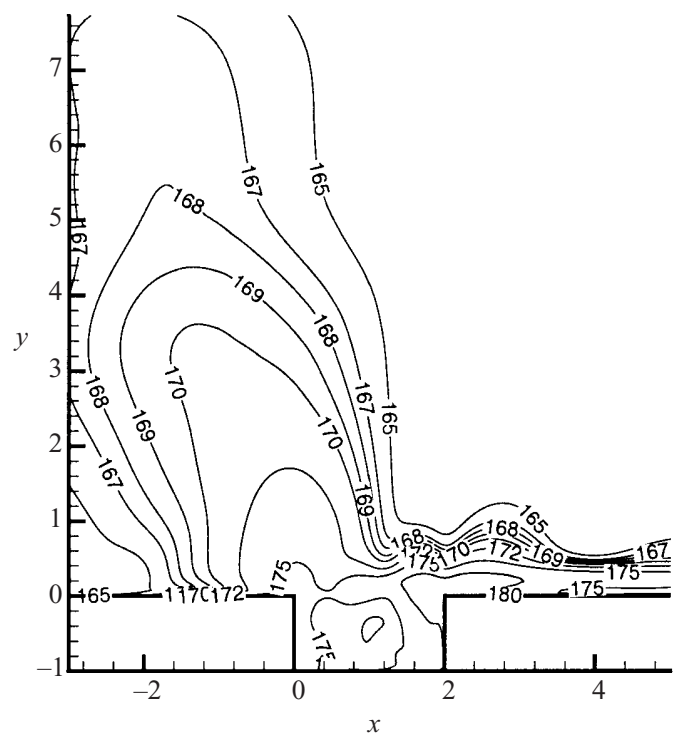

FIGURE 7. Sound pressure level (SPL) for run L2.

downstream axis. Sound pressure levels are still at about $170 \mathrm{~dB}$ at 3 cavity depths along the peak radiation direction. These numbers are quite high, but Krishnamurty (1956) estimated sound pressure levels for the case of laminar boundary layers in excess of $163 \mathrm{~dB}$ for a variety of geometries. These estimates were based on deflections from finite-fringe interferometry, but no detailed mapping to SPL was performed. Krishnamurthy (1956) found that laminar boundary layers upstream produce higher SPL than their turbulent counterparts, and that the $L / D=2$ cavity was louder than longer cavities.

Figure 8 illustrates the separate contributions to the acoustic field when two resonant frequencies are present simultaneously. Density fluctuations are plotted for run $L 2$, which has two strong resonant frequencies, at $S t=f L / U=0.4$ and 0.7 . We performed a discrete Fourier transform (DFT) of 125 samples (every 250 time units) of the computational data over a period of time $T U / L=30$, corresponding to 12 periods of the lower frequency, and 21 periods of the higher frequency. The resulting data record is approximately periodic in time, and any drift in the data is removed prior to taking the DFT. We experimented with different windowing techniques, signal durations, and sampling rates, and we believe the results presented here are free from any artifacts of the limited signal duration and sampling rates.

Real and imaginary parts of the DFT are plotted, and the different wavelengths of far-field acoustic radiation are apparent. Hydrodynamic disturbances in the shear layer are also evident, and will be discussed further in §3.4. It is striking that the level of the density fluctuations in the far field is comparable to the level of the density fluctuations in the shear layer. This indicates the high efficiency of the acoustic scattering process at the cavity trailing edge. The wavelength of the acoustic fields at differing angles into the free stream can be measured, and is in agreement with the predicted value based on the frequency of oscillation and the speed of the wavefront, $a_{\infty}(1+M \cos \theta)$, where $\theta$ is measured from the downstream $x$-axis, and $a_{\infty}$ is the ambient sound speed. 

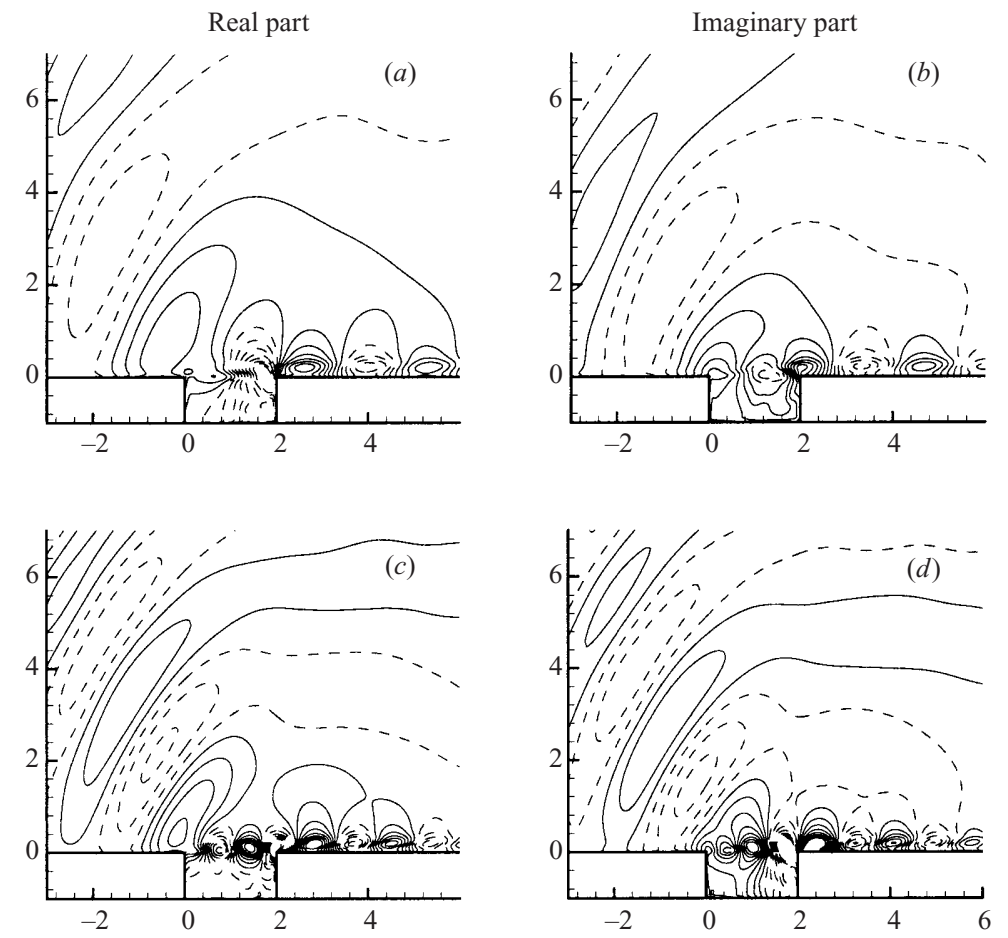

Figure 8. Contours of the DFT of the density at the two resonant frequencies for run L2. $(a, b)$ 16 levels between $\pm 0.008 \rho_{\infty}$ for $S t_{1}=0.4 ;(c, d) 16$ levels between $\pm 0.004 \rho_{\infty}$ for $S t_{2}=0.7$; negative contours are dashed.

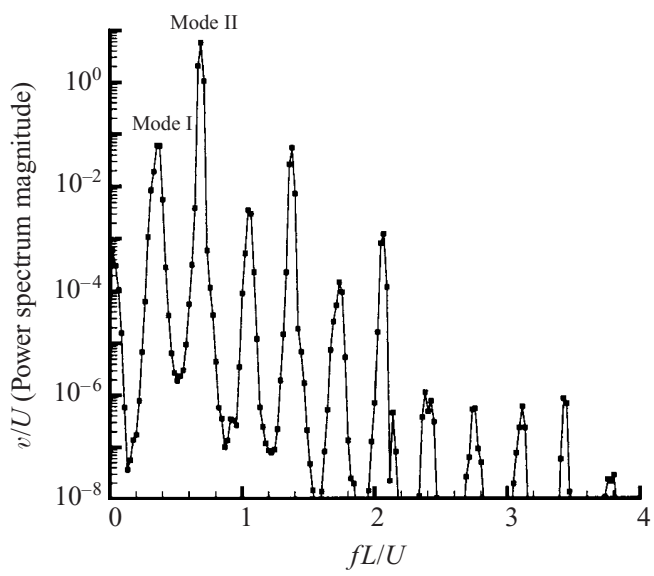

FigURE 9. Spectrum of normal velocity at $y=0$ and $x=1.57 D$, for run $2 \mathrm{M} 7$.

\subsection{Frequencies of oscillation}

Figure 9 shows the spectrum of normal velocity at a point along the cavity mouth, at $y=0, x / L=0.783$, for a $L / D=2$ cavity with $M=0.7$ (run 2M7), which is oscillating in the shear-layer mode. Several distinct peaks and their harmonics are evident. The frequencies labelled 'Mode I, II' correspond to the first two frequencies 


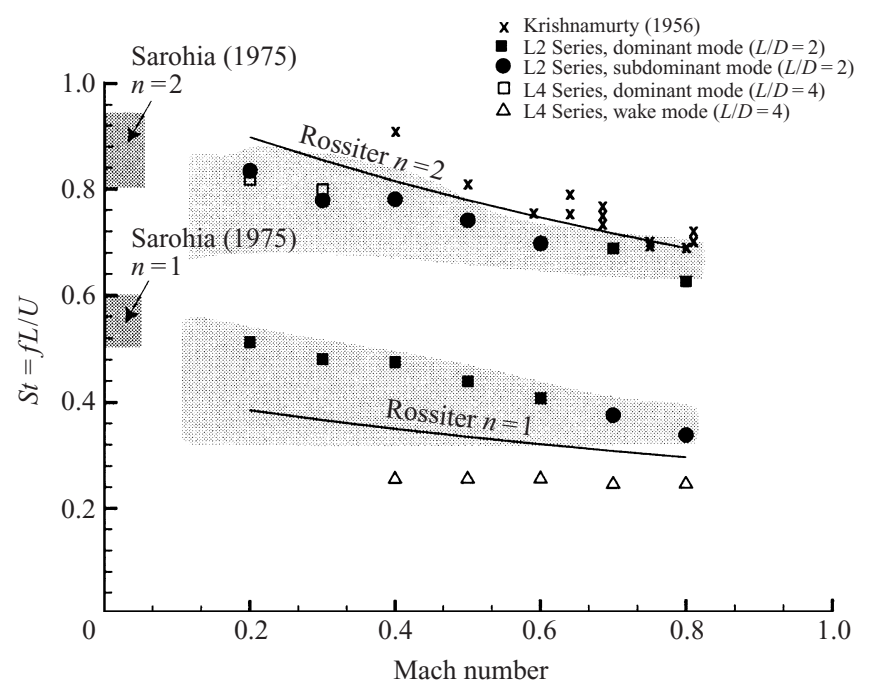

FIGURE 10. Strouhal numbers for peaks in spectra for the $2 \mathrm{M}$ series of runs (shear-layer mode), and 4M series (shear-layer and wake modes) compared to experiment and equation (3.1). The lighter shaded region indicates the scatter of data in experiments with turbulent boundary layers by Heller \& Bliss (1975), Rossiter (1964) and Tam \& Block (1978), and the darker shaded region indicates the frequencies measured by Sarohia (1975).

predicted by Rossiter's semi-empirical formula

$$
S t_{n}=\frac{f_{n} L}{U}=\frac{n-\gamma}{M+1 / \kappa}, \quad n=1,2, \ldots,
$$

where $S t_{n}$ is the Strouhal number corresponding to the $n$th mode frequency $f_{n}$, and $\kappa$ and $\gamma$ are empirical constants corresponding to the average convection speed of disturbances in the shear layer, and a phase delay. The other peaks in figure 9 are either harmonics or nonlinear interactions between the Rossiter modes.

In figure 10 , the frequencies of the two most energetic peaks in the spectra for the series of runs $2 \mathrm{M}$ with $L / D=2$ and $L / \theta_{0}=52.8$ and $4 \mathrm{M}$ with $L / D=4$ and $L / \theta_{0}=102$ are compared to experimental data and predictions from equation (3.1), with $\gamma=0.25$ and $\kappa=1 / 1.75$, the original values used by Rossiter. Krishnamurty (1956), whose conditions match the DNS most closely, detected only one mode in experiments with laminar boundary layers upstream, and the frequencies are somewhat higher than mode 2 oscillations from the DNS. As noted above, the Reynolds number in his experiments is about 5 times that of the DNS. Sarohia (1975) has shown that for low Mach number the frequency of oscillation increases as the Reynolds number is increased. He found frequencies for modes 1 and 2 at low Mach number as shown in figure 10, and it would appear that the frequencies of oscillation at higher Mach numbers increase smoothly to the case $M=0$. Meanwhile, experimental data for turbulent boundary layers, the scatter of which is shown by the lighter shaded regions on the plot (Tam \& Block 1978; Heller \& Bliss 1975; Rossiter 1964), tend to fall somewhat below the laminar case, as noted previously (Krishnamurthy 1956; Sarohia 1975).

There is quite a lot of scatter in the frequencies measured in experiments at low Mach numbers. In general, the data from experiments in water are fairly consistent (Sarohia 1975; Knisely \& Rockwell 1982), and the darker shaded region in figure 10 
indicates the frequencies measured by Sarohia. Experiments in air at low Mach numbers have more scatter (Tam \& Block 1978; Rockwell \& Schachenmann 1982; Ahuja \& Mendoza 1995), because the frequencies of the resonant acoustic modes of the cavity itself become comparable to the Rossiter frequencies.

Peaks in the spectra corresponding to harmonics and sum and difference modes (primarily harmonics of $n=1$ and the sum of Rossiter modes $n=1$ and $n=2$ ) were also detected in the data. Such nonlinear interactions between modes were studied by Knisely \& Rockwell (1982) for incompressible cavity flow, and more recently by Cattafesta et al. (1998) for moderate subsonic speeds, with turbulent upstream boundary layers. These studies used bicoherence analysis and other sophisticated signal processing tools that rely on long time series of data. For the spectra obtained here, signal durations were relatively short, on the order of 20-100 times the lowest frequency peak in the data, so it was difficult to detect the presence of any mode switching, as was found to occur over long times by Cattafesta et al. (1998). In the computations, it appears that the two primary Rossiter modes $(n=1,2)$ were coexistent.

Finally, we note that in figure 10 , oscillation frequencies from the $4 \mathrm{M}$ series of modes show the transition to wake mode oscillations for $M>0.3$. These data are discussed more fully in $\S 4$.

\subsection{Shear-layer spreading rate}

The shear layer spanning a cavity differs from a mixing layer in two main respects: Kelvin-Helmholtz instabilities are constantly being excited by the intense acoustic environment; and the entrainment is modified by the presence of the cavity. Despite these differences, most researchers report that shear layers over cavities closely resemble turbulent free shear layers, in that the spreading rate is approximately linear. However, there is some discrepancy in the actual value of the spreading rate.

Here, we use the vorticity thickness $\delta_{\omega}=U /(\mathrm{d} U / \mathrm{d} y)_{\max }$ as a measure of the shearlayer thickness. It is common to use the momentum thickness $\theta$, defined by integrating the flux of the momentum deficit from the cavity bottom to infinity. However, the momentum thickness shows significant variation caused by the recirculating region in the downstream portion of the cavity. The vorticity thickness is a local measure of the maximum shear, which better determines instability properties of the shear layer, and so it is the thickness we choose.

Sarohia (1975) appears to have been the first to measure the spreading rate in detail, and found that the spreading was approximately linear, and the spreading rate increased as $L / \theta_{0}$ increased. As $L / \theta_{0}$ increased from 52.5 to 105.2 , with $R e_{\theta}$ and $D \theta_{0}$ held constant, the spreading rate $\mathrm{d} \delta_{\omega} / \mathrm{d} x$ varied from 0.025 to 0.088 . These values are significantly lower than typical values for turbulent free shear layers, generally accepted to be around $\mathrm{d} \delta_{\omega} / \mathrm{d} x \approx 0.162$ (Brown \& Roshko 1974). The upstream boundary layers in Sarohia's experiments were laminar. Gharib \& Roshko (1987) also found linear growth of the shear layer, and found that the spreading rate was fairly constant at $\mathrm{d} \delta_{\omega} / \mathrm{d} x=0.124 \uparrow$ for cavities with $L / \theta_{0}>103$. This indicates a spreading rate much closer to that of a turbulent free shear layer. The recent lowMach-number experiments of Cattafesta et al. (1997) also exhibit linear spreading, with turbulent upstream boundary layers. Though the spreading rate is not stated, they report that for a cavity with $L / \theta_{0}=328$, the spreading rate closely matches that

$\dagger$ Where thicknesses are given in terms of momentum thickness $\theta$, we set $\delta_{\omega}=4 \theta$, which is exact for a hyperbolic tangent profile. 


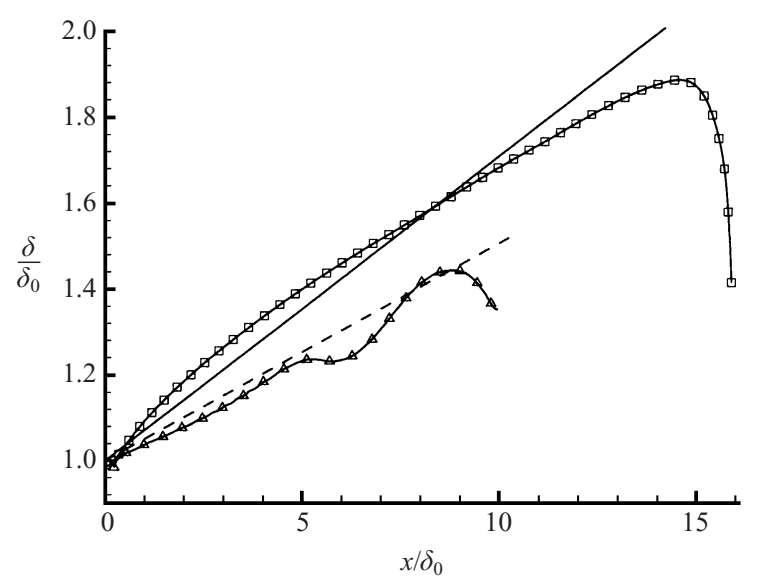

FIGURE 11. Vorticity thickness $\delta_{\omega}$ along the shear layer for runs L2 $(\triangle)$ and LG6b ( $\square$ ), and linear fits with slope $\mathrm{d} \delta_{\omega} / \mathrm{d} x=0.05(---)$ and $0.07(-)$.

of a turbulent free shear layer, but for a cavity with $L / \theta_{0}=81$, the spreading rate is $50 \%$ higher, the opposite trend to that observed by Sarohia.

Shear-layer thicknesses from two of our runs are plotted in figure 11. Our data also indicate approximately linear growth, with spreading rates similar to those measured by Sarohia. Our run L2 $\left(L / \theta_{0}=53\right)$ has a spreading rate of about $\mathrm{d} \delta_{\omega} / \mathrm{d} x=0.05$, while Sarohia's experiments had $\mathrm{d} \delta_{\omega} / \mathrm{d} x=0.056$ for $L / \theta_{0}=60$. Our spreading rates also increase with $L / \theta_{0}$, and for run LG6b $\left(L / \theta_{0}=90\right)$ we measure $\mathrm{d} \delta_{\omega} / \mathrm{d} x=0.07$, compared to an experiment of Sarohia's with $L / \theta_{0}=85$ and $\mathrm{d} \delta_{\omega} / \mathrm{d} x=0.064$. Thus, our measurements support the trend noted by Sarohia, that the shear layer spreads faster for longer cavities. A likely reason for this trend is that a longer shear layer amplifies disturbances more, so the final amplitude of oscillations is larger, thus increasing Reynolds stresses, which cause the spreading.

\subsection{Convection and amplification by the shear layer}

In this section, we examine how disturbances are convected and amplified by the shear layer, and we compare the DNS results to those predicted by linear stability theory. The linear stability calculations are performed in a standard way, and will be described only briefly here. We use a compressible, inviscid, locally parallel formulation, and thus we assume that velocity profiles are slowly varying in the streamwise direction, but that at each streamwise location the flow is parallel. Normal modes of the form

$$
f(x, y, t)=\hat{f}(y) \mathrm{e}^{\mathrm{i}(\alpha x+\omega t)}+\text { c.c. }
$$

are assumed, where c.c. denotes the complex conjugate. For spatial instability, $\omega$ is the real frequency, $\alpha$ is the complex wavenumber, $f$ is any flow variable, and $\hat{f}(y)$ is the complex eigenfunction. These modes are inserted into the inviscid, compressible equations linearized about the parallel flow (for the flow speeds and isothermal wall conditions considered here, the mean density is nearly uniform, and for simplicity we ignore the slight density variation in the stability calculation), and the eigenvalues and eigenfunctions are found using an adaptive Runge-Kutta shooting method. Boundary conditions are that the disturbances are exponentially damped for large $+y$, and zero normal velocity is imposed at the cavity bottom. We note that in previous analyses 


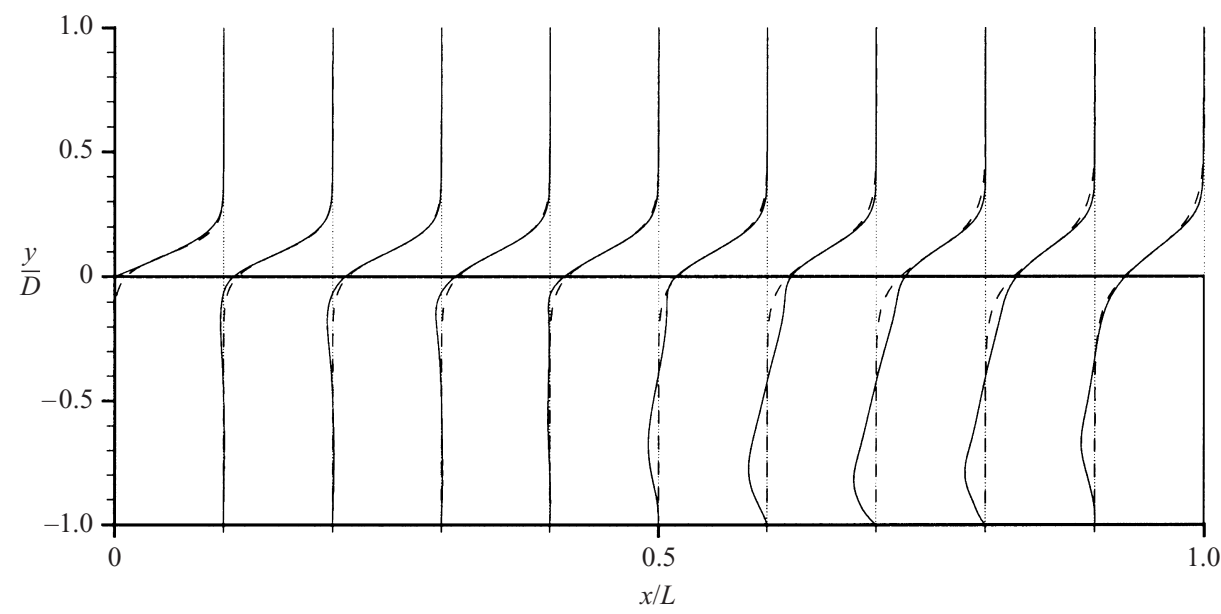

FIGURE 12 . Mean streamwise velocity profile, $\bar{u}(x, y) / U$, for run L2 ( - ) compared to the hyperbolic tangent profiles with the same vorticity thickness $(----)$.

(e.g. Tam \& Block 1978) the cavity bottom is not accounted for, as the cavity was assumed infinitely deep compared to the shear-layer thickness.

We use the simulations to determine the mean flow for the linear stability calculations. Two different calculations are performed, one using the actual velocity profiles from the DNS, and another using hyperbolic tangent profiles with the same vorticity thickness and deflection. As shown in figure 12, the agreement is good close to the cavity leading edge, but much worse near the rear of the cavity, where the steady captive vortex is present. The actual DNS velocity profiles will of course be more accurate, but the tanh profiles yield very similar results, and later (§5), we use the tanh-profile linear stability calculations as a predictive tool, to predict the total amplification by the shear layer.

The overall magnitude and phase of the instability wave is found by integrating the complex growth rate $\alpha$ over the streamwise positions:

$$
\tilde{f}(x, y)=\hat{f}(y) \exp \left(\mathrm{i} \int_{0}^{x} \alpha \mathrm{d} x\right),
$$

where both the wavenumber $\alpha$ and the eigenfunction $\hat{f}$ are slowly varying functions of $x$. Without carrying the analysis to higher order, the normalization of the eigenfunctions is arbitrary, and we have set the maximum amplitude of the streamwise velocity mode to unity at each axial position.

Figure 13 shows the normal velocity instability waves $\tilde{v}$, at the two resonant frequencies present in run L2 $(S t=0.4$ and 0.7$)$, using the mean flow from the same run. Also shown is the DFT of the normal velocity, computed as described in $\S 3.1$.

The linear stability eigenfunctions are seen to be in very good qualitative agreement with the Kelvin-Helmholtz instability waves at the corresponding frequencies, except very near the cavity trailing edge, where the DNS results show some of the disturbances being swept down into the cavity by the (nearly steady) vortex that occupies the downstream half of the cavity.

A more quantitative measurement of the phase variation is presented in figure 14 . The phase of vorticity disturbances in the shear layer is plotted, again for run L2, at the two resonant frequencies. (This is just the phase of the DFT of vorticity, along $y=0$.) 


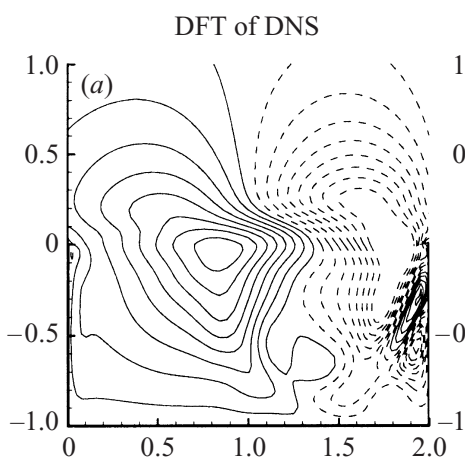

LS, DNS velocity profile

LS, tanh velocity profile
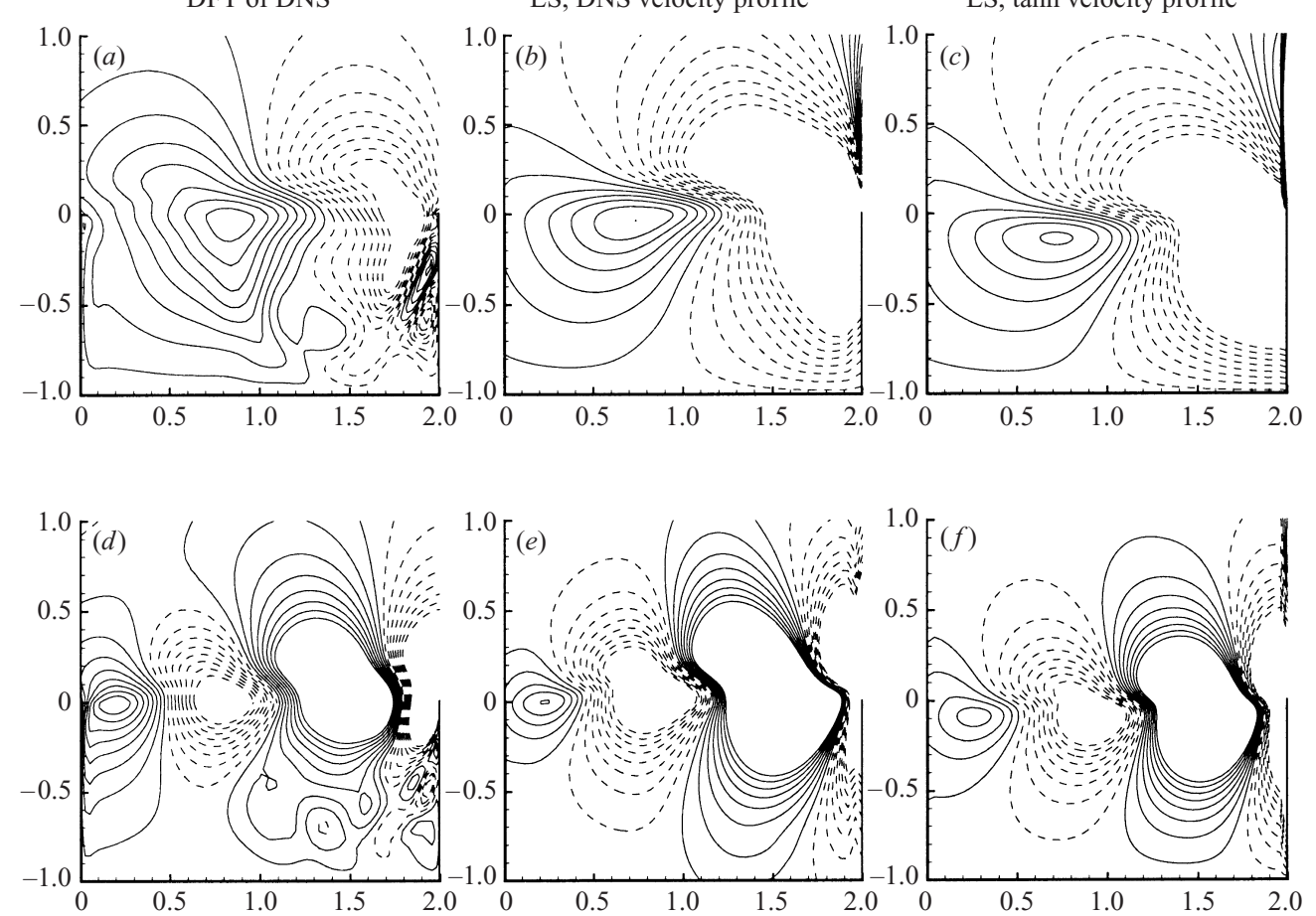

FIGURE 13. Comparison of mode shapes (real part) for normal velocity fluctuations in the shear-layer region for parameters of run L2. Contours are between $\pm 0.01 U$ for $S t_{1}=0.4$ in $(a-c)$, between $\pm 0.005 U$ for $S t_{2}=0.7$ in $(d-f)$.

Also plotted is the phase of the dilatation close to the cavity floor, which represents the upstream-travelling acoustic wave inside the cavity. These variables were chosen to better separate hydrodynamic disturbances from acoustic disturbances, though very similar results were obtained when normal velocity and pressure were used in the shear layer and cavity floor, respectively. The dilatation phase is relatively constant in the $y$-direction, except near the shear layer, where the hydrodynamic fluctuations are significant. Notice that the total phase variation from the shear-layer convection (downstream) and acoustic propagation (upstream) is almost exactly $2 \pi n$, where $n$ is the index of the Rossiter mode. This phase criterion is similar to that found in several experiments (Knisely \& Rockwell 1982; Rockwell \& Schachenmann 1982; Gharib \& Roshko 1987), which show that in the low-Mach-number limit, the total phase variation in the shear layer alone is a multiple of $2 \pi$. In this limit, the acoustic propagation is of course instantaneous, so for our compressible simulations we must add the phase variation of the finite-speed acoustic propagation. Note that the phase speed of the acoustic waves along the cavity bottom is far from constant. This indicates the presence of multiple acoustic reflections by the cavity walls.

Linear stability predictions for the phase variation in the shear layer are also plotted, and the phase variation is predicted well except for very near the leading and trailing edges of the cavity, where the DNS data show a slower phase speed (steeper phase variation). We do not expect the linear stability calculations to be very accurate in these regions, because the flow is rapidly changing in the streamwise direction, and significantly non-parallel at the downstream corner. In addition, any flow/acoustic 


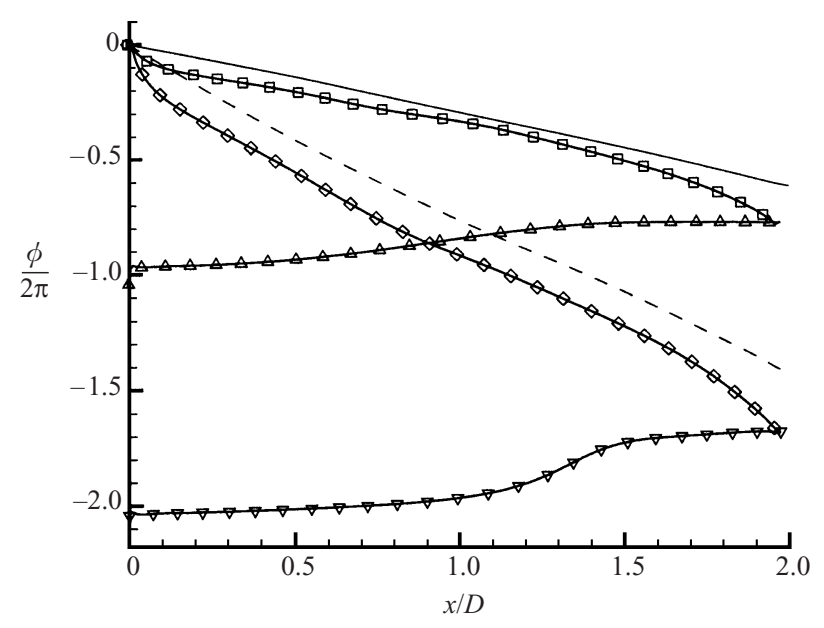

FIGURE 14. Phase of vortical and acoustic disturbances for run L2, at the frequencies of the first two Rossiter modes $(S t=0.4,0.7)$. Vorticity disturbances in the shear layer at $y=0(\square, \diamond)$; dilatation along cavity bottom at $y=-0.99 D(\triangle, \nabla)$; and shear-layer phase predicted by linear stability $(-$, $---)$. Dilatation phase is shifted to line up with vorticity phase at $x=L$.

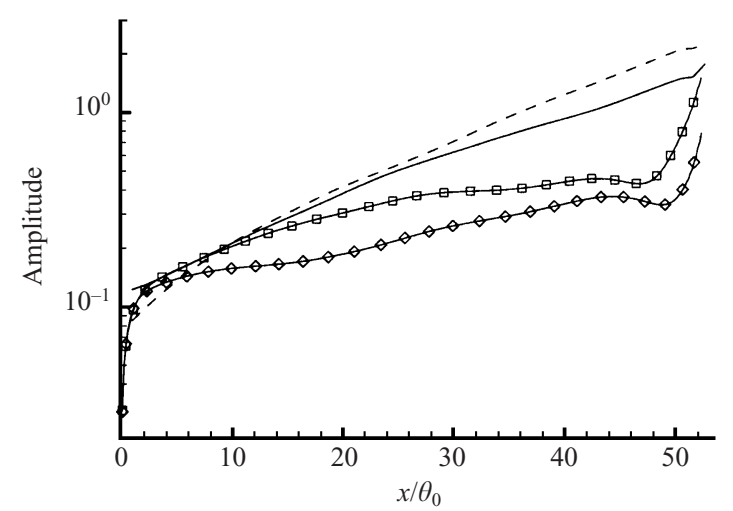

Figure 15. Amplitude of normal velocity fluctuations (run L2) along $y=0$ at $S t_{1}=0.4$ ( $\square$ ) and $S t_{2}=0.7(\diamond)$; and predictions from linear stability for $S t_{1}(-)$ and $S t_{2}(----)$.

coupling will be most important near the cavity corners, where the receptivity to acoustic disturbances is high. These effects have been studied in detail by Rockwell \& Schachenmann (1982).

Recall that Rossiter's formula for the resonant frequencies includes an empirical constant $(\gamma$ in equation (3.1)) that represents an additional phase lag somewhere in the feedback loop. It is possible that the steeper phase variation exhibited near the leading and trailing edges of the cavity is the cause of this additional phase variation. The average phase speed from the DNS data is $c_{p} / U=0.49$ for $S t=0.4$ and $c_{p} / U=0.41$ for $S t=0.7$, while the linear stability calculations predict phase speeds of 0.63 and 0.49 for $S t=0.4$ and 0.7 .

Figure 15 shows the amplitude of normal velocity disturbances in the shear layer. The measured growth rates are significantly smaller than those predicted by the linear theory, which is surprising, because several experiments show the amplitude 

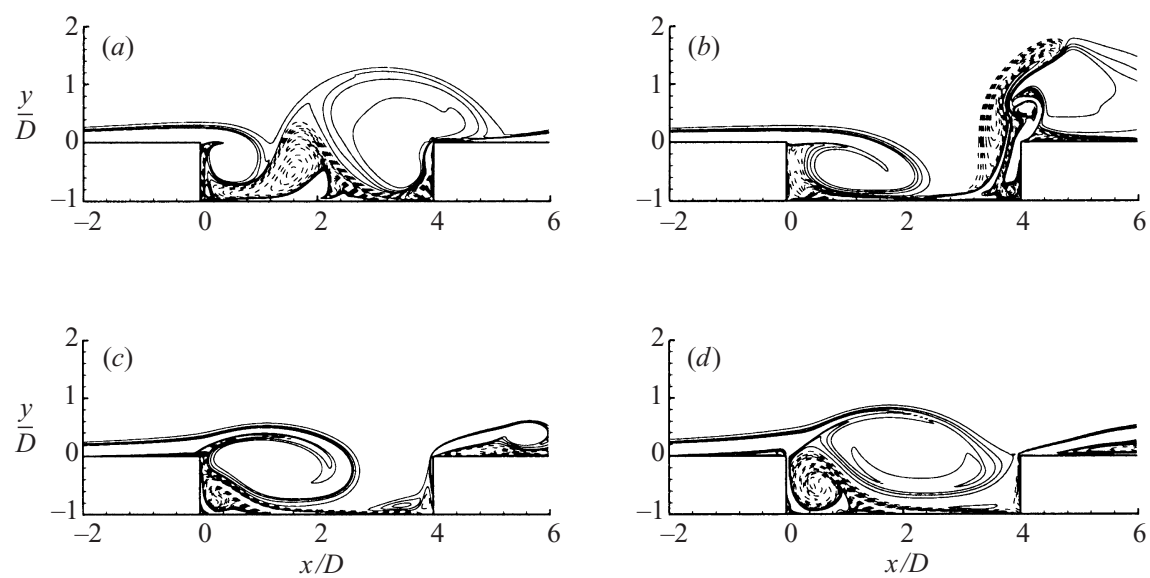

FigURE 16. Instantaneous vorticity contours for run L4 (wake mode) at four different times ( $a-d$ ), corresponding to approximately quarter-phase intervals of the periodic cycle. 15 contours between $\omega D / U=-5$ and 1.67. Positive contours are dashed. Only a small portion of the computational domain near the cavity is shown.

is predicted well, at least for moderate values of $x / \theta$. Knisely \& Rockwell (1982) used a constant-thickness mean profile, and found that the amplitude matched the linear theory well, for $x / \theta_{0} \leqslant 30$; Cattafesta et al. (1997) found good agreement for $x / \theta_{0} \leqslant 60$, also using a constant-thickness mean profile. However, our Reynolds number is much smaller than that in either of these experiments, so presumably a viscous stability calculation would agree better.

In summary, linear stability theory gives reasonable predictions for the mode shapes of the resonant frequencies, and also the convection speeds of disturbances, but amplification rates are significantly over-predicted. The linear stability calculation was compressible, but inviscid, and locally parallel. Adding viscous effects, including effects of flow/acoustic coupling, or carrying out a multiple scales analysis to account for slightly non-parallel effects (e.g. Crighton \& Gaster 1976) may provide a better agreement than is obtained here.

\section{Wake mode}

As the length or depth of the cavity (relative to the upstream boundary-layer thickness) and/or Mach and Reynolds numbers is increased, there is a substantial change in the behaviour of the cavity oscillations. Under these conditions, the flow is characterized by a large-scale shedding from the cavity leading edge. As noted in the introduction, Gharib \& Roshko (1987) were the first to understand this transition in detail, and used the term wake mode to describe the resulting flow regime. Connections with the experiment are discussed further below. The shed vortex has dimensions of nearly the cavity size, and as it is forming, irrotational free-stream fluid is directed into the cavity, impinging on the cavity base. The vortex is shed from the leading edge and ejected from the cavity in a violent event. The vortex is large enough to cause flow separation upstream of the cavity during its formation, and again in the boundary layer downstream of the cavity as it convects away. Figure 16 shows four snapshots of the vorticity field in wake mode for run L4 over one period of oscillation. 


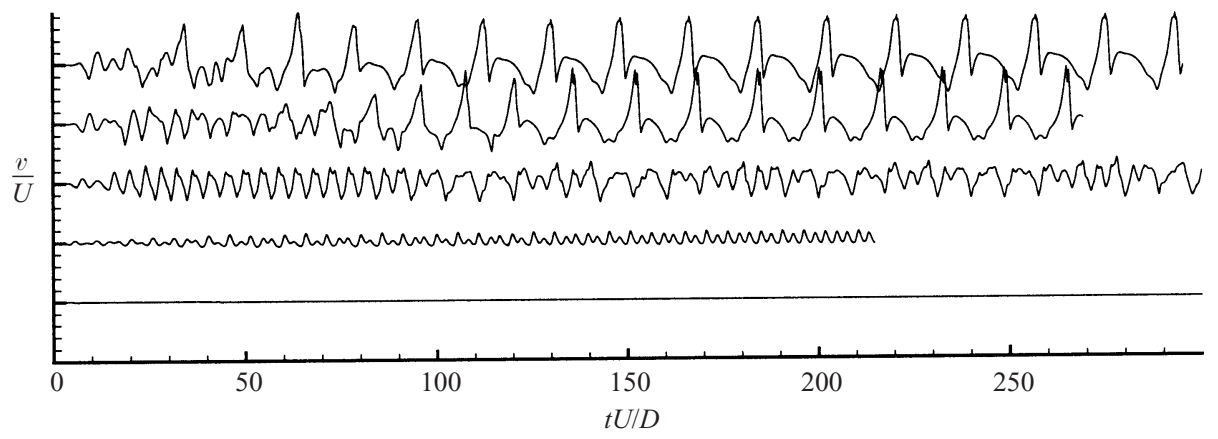

Figure 17. Time traces of the normal velocity, relative to $U$, at $y=0, x=3.13 D$ for the series of runs L1-L5 (bottom to top) The vertical axes have been artificially shifted to show all the data clearly, with major tick marks representing 1 unit.
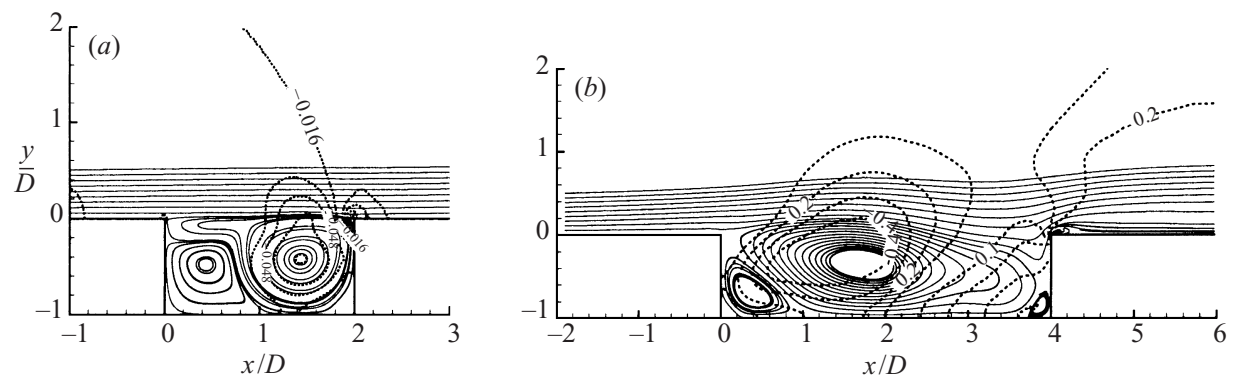

FiguRE 18. Time-averaged flow for (a) L2 (shear-layer mode), and (b) 4M6 (wake mode). Mean streamlines (solid lines) are superposed on contours of constant $C_{p}$ (dashed lines).

\subsection{Flow features}

Time traces of the normal velocity at $y=0$ and $x=3.13 \mathrm{D}$ are shown in figure 17 , for the series of runs L1-L5, where $L / \theta_{0}$ was varied, with constant $D / \theta_{0}$. It is evident that the transition from shear-layer mode to wake mode occurs as $L / \theta_{0}$ is increased through 75. For $L / \theta_{0}=25$, the oscillations are damped and the flow becomes steady. For $L / \theta_{0}=75$, it appears that there is mode switching, with wake and shear-layer modes being present at different times (what we referred to in table 1 as the mixed mode). The transition is also a function of $M$, and for $L / \theta_{0}=102$, the shear-layer mode exists for $M<0.3$ and the wake mode for $M>0.3$. Again, time traces for flows near the transition indicated the presence of mode switching.

Figure 18 contrasts the time-averaged flow for runs L2 (shear-layer mode) and L4 (wake mode). The mean streamlines in the wake mode are significantly deflected above the cavity, and show that on average the boundary layer upstream of the cavity sees an adverse pressure gradient. On average the flow in the cavity is strongly recirculating, and there is an impingment of the recirculating flow on the rear wall. It is important to contrast the mean flow with the instantaneous visualizations of figure 16, which shows there is no stationary vortex within the cavity instantaneously. The region of high pressure near the back corner of the cavity resembles that observed by Fox (1965) in his high-drag flow regime. Variations in the average coefficient of pressure are also quite large, reaching a minimum of about -0.5 where the flow is expanding into the cavity, to about 0.3 in the impingment region on the rear step. 


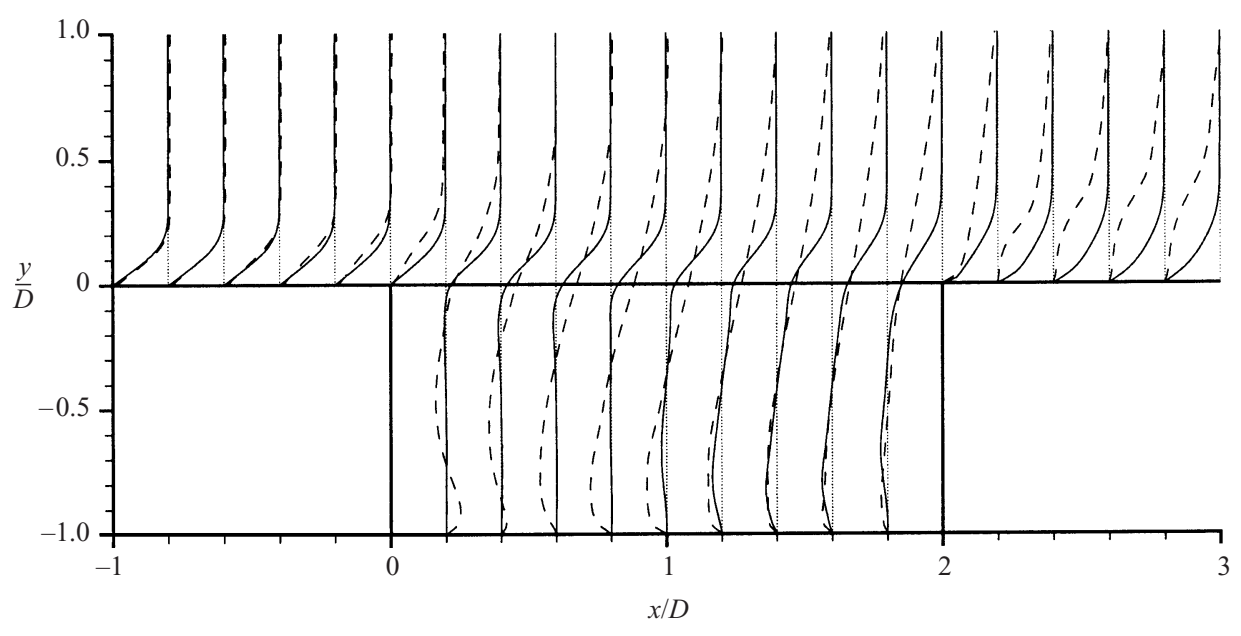

FIGURE 19. Mean streamwise velocity profiles, $\bar{u}(x, y) / U$, at different streamwise positions. Run L2 ( - ; Run L4 (----).

By contrast, the shear-layer mode shows much smaller pressure variations, and mean flow streamlines are nearly horizontal along the mouth of the cavity. The low pressures $\left(C_{p} \approx-0.08\right)$ correspond to the centre of the recirculation region that exists in the rear two-thirds of the cavity (see figure 4). The low-pressure region appears to be the result of the vortical swirling motion in the cavity, and not because of an expansion of the flow into the cavity as was the case for the wake mode. There is a very small impingment region at the rear edge, where $C_{p}$ reaches about 0.1 . However, over the majority of the rear face $C_{p}<0$, which is consistent with the measurements of Roshko (1955) and others.

Similar flow features may be seen in mean streamwise velocity profiles, which are shown for both modes in figure 19. It is again evident that the oscillations in wake mode have a substantial impact on the mean flow as far as 1-2 depths above the cavity. The influence of the wake mode is, in the mean, significant to about a quarter of a depth upstream of the cavity. As we noted in $\S 2$, the momentum thickness at the cavity edge that we have quoted is that of the initial condition, and figure 19 shows that this is indeed substantially modified by the flow in wake mode (it is modified only by a very small amount in the shear-layer mode).

In Gharib \& Roshko (1987), transitions between non-oscillatory, shear-layer, and wake modes occurred at $L / \theta_{0}=80$ and $L / \theta_{0}=160$, respectively. The present data indicate a change from wake mode to shear-layer mode that depends also on the Mach number and Reynolds number. The specific parametric dependence of the transition to wake mode is discussed in $\S 4.3$. The drag on the cavity (given for selected runs in table 1), is of comparable magnitude to the values reported by Gharib \& Roshko (1987) for the different regimes. An impingment of the flow on the rear step was also noted in the experiments, and it also appears (see figure 7(a) of Gharib \& Roshko 1987) from dye visualizations that the boundary layer separates upstream of the cavity leading edge.

\subsection{Frequency of vortex shedding}

The spectra of the resonant instabilities in wake mode are very different from those of the shear-layer mode (§3.2). After an initial transient, which at early times is similar 


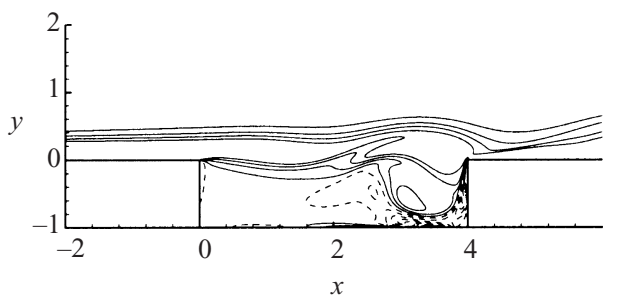

FIGURE 20. Instantaneous vorticity contours for run TK4b (shear-layer mode). Contour levels same as figure 16.

to shear-layer mode, the flow becomes nearly periodic in time, with the fundamental period corresponding to the vortex shedding from the leading edge (see figure 17). The spectrum (after the onset of wake mode) consists of a dominant frequency and strong peaks at its harmonics. The $4 \mathrm{M}$ series of runs transitioned from shear-layer to wake mode for $0.3<M<0.4$, and the peak frequencies were plotted in figure 10 . For $M>0.3$, the peaks fall well below the first Rossiter mode prediction, and, unlike the shear-layer mode, they show little variation with $M$. For $M=0.4$ to $M=0.8$ the fundamental frequency varied less than $4 \%$, compared to the expected variation of about $20 \%$ for Rossiter mode one. The $4 \%$ variation is, in fact, within the uncertainty associated with the total sampling period used to determine the frequency.

The lack of variation with $M$ indicates that the wake mode is not a result of acoustic feedback, and it appears that the feedback in this case is provided by the complicated recirculating flow in the cavity. This is discussed further in $\S 4.4$.

\subsection{Parametric dependence of the transition to the wake mode}

In table 1 , we have indicated the dominant mode (steady flow, shear-layer mode, or wake mode) for the different runs, and, for the wake mode cases, the shedding frequency $S t_{D}=f D / U$. (Note that here the cavity depth is used for the nondimensionalization.) Once established, the shedding frequencies in wake mode are all nearly the same for cavities with $L / D=4$, meaning that, in addition to the invariance to Mach number noted above, they are not influenced by $L / \theta_{0}$ or $D / \theta_{0}$. A lack of dependence of the shedding frequency on the boundary-layer thickness is a common feature of vortex shedding behind bluff bodies (for instance, for laminar flow over a cylinder, it is well known that the shedding frequency $S t_{D}=f D / U$ is constant over a wide range of Reynolds numbers). We note that for the cavity with $L / D=5$ (run L5), the shedding frequency is about $10 \%$ lower than that of the $L / D=4$ runs, and that a periodic wake mode was not detected in any of the runs with $L / D=3$ or smaller. Several of the $L / D=3$ runs exhibited some characteristics of both shear-layer mode and wake mode.

While the wake mode typically occurs for larger $L / \theta_{0}$ and $D / \theta_{0}$, the transition depends as well on the Mach and Reynolds numbers. For example runs L4 and TK4b both have $L / D=4$, but the latter is oscillating in shear-layer mode, as shown by the instantaneous vorticity fields plotted in figure 20 , which should be contrasted to figure 16. Moreover, the transition to the wake mode also depends on $M$, but it is interesting that, as previously noted, once the wake mode is established the frequency of vortex shedding is nearly independent of $M$. The $4 \mathrm{M}$ series of runs shows transition to the wake mode as the Mach number is increased, holding other parameters constant. The TK4 series of runs shows evidence of transition to wake mode as $D / \theta_{0}$ is increased, holding $L / D, M$ and $\operatorname{Re}_{\theta}$ nearly constant, and, finally, 
the R4 series shows transition with increasing $\operatorname{Re}_{\theta}$ when $L / D, M$ and $D / \theta_{0}$ are held nearly constant.

In all of the simulations that have been performed, examination of the instantaneous flow fields shows that the initial growth of instability is always in shear-layer mode, where acoustic radiation from the downstream edge is the mechanism for the feedback. The shear-layer oscillations, at early times, are growing in amplitude with each subsequent cycle, and the wake mode sets in only after the oscillations have reached a certain threshold. Thus it appears that the emergence of the wake mode is determined by the conditions in the shear-layer mode, and this forms the basis of our attempt below to model the parametric dependence of the wake mode. We note that we have not attempted to look for hysteresis in the transition to wake mode, as there is no unambiguous way to do so computationally.

\subsection{Convective and absolute instability}

As indicated above, the oscillation frequency in the wake mode is independent of the Mach number, over the large range $0.4<M<0.8$. This implies that, unlike the oscillations described by the Rossiter mechanism, wake mode oscillations are not acoustically forced convective instabilities. In the wake mode, the flow exhibits several qualitative features of absolutely unstable parallel flows, as described in, e.g. Huerre \& Monkewitz (1985), and this may provide an explanation of the governing mechanism.

The notion of absolute or convective instability is a useful tool for describing selfsustained oscillations in parallel shear flows. The idea is the following: given a certain parallel flow, if energy from a disturbance grows in time, but travels only downstream, the flow is called convectively unstable. In such flows, there is no possibility for selfsustained oscillations, since if the flow disturbance is removed, the oscillations convect away. However, if energy grows and travels upstream, then the flow is called absolutely unstable, and such flows have the potential for self-excitation. In this context, flows that exhibit self-sustained oscillations are called globally unstable (Huerre \& Monkewitz 1990). This terminology is normally reserved for (nearly) parallel flows, however - nonparallel flows will normally not be steady solutions of the Navier-Stokes equations, so it does not make sense to discuss their stability.

For cavity oscillations in the shear-layer mode, the flow is nearly parallel, so these stability concepts apply directly. The established view of cavity oscillations in the shear-layer mode is one of a convective Kelvin-Helmholtz instability in the shear layer, which leads to global instability through pressure feedback from acoustic waves generated near the trailing edge. Indeed, if $D / \theta_{0} \gg 1$, so that the boundary condition at the cavity bottom may be neglected, and if flow within the cavity is relatively quiescent (such that the velocity ratio across the shear layer is approximately unity), then analysis shows that the shear layer is indeed (locally) convectively unstable (Huerre \& Monkewitz 1985). The good prediction of oscillation frequencies by the Rossiter formula, and the computational confirmation given earlier (e.g. figure 5 and the results of §3.4), confirm the feedback process leading to global instability.

In the wake mode, the flow past the cavity is far from parallel, and the disturbances are far from small, so the notions of absolute and convective stability do not strictly apply. Nevertheless, these notions have been applied to very similar oscillating wake flows, such as the wake behind a cylinder, and the predictions of the theory have been shown to be in good agreement with experiment, despite these non-parallel, largeamplitude effects (Huerre \& Monkewitz 1990). We therefore apply these ideas to the cavity flow in wake mode, even though the theoretical justification is questionable.

With these ideas in mind, the flow in wake mode is also globally unstable, since self- 
sustained oscillations persist despite no external forcing. However, since the frequency is independent of Mach number, the feedback mechanism is apparently no longer acoustic. It is therefore plausible that absolute instability provides the mechanism necessary for global instability.

The mean flow profiles shown in figure 19 show that in shear layer mode (run L2), there is very little backflow in the cavity, and furthermore the backflow is confined to a region at the rear of the cavity. By contrast, the wake mode profiles (run L4) reveal a substantial backflow even very close to the cavity leading edge. Huerre \& Monkewitz (1985) have shown that for tanh profiles with greater than $13.6 \%$ backflow, the flow is absolutely unstable. The backflow in the mean profiles for run L4 reaches a maximum of $38 \%$ of the free-stream velocity near the centre of the cavity, decreasing to about $21 \%$ within two boundary-layer thicknesses of the leading edge, and $15 \%$ within one boundary-layer thickness. The backflow for run L2 is much smaller, reaching a maximum of $23 \%$ of the free-stream near the rear of the cavity, and never exceeding $3 \%$ of the free-stream anywhere in the front $40 \%$ of the cavity. The mean profiles from run L4 are not described well by tanh profiles, but the main point is that there is significant backflow in the cavity, and in this sense the profiles are qualitatively similar to profiles which have been shown to be absolutely unstable. It is possible, then, that absolute instability may provide a mechanism for transition to wake mode. It is also conceivable that similar ideas to those expressed in Monkewitz \& Nguyen (1987) might be used to predict the shedding frequency in wake mode.

\section{Prediction of the oscillation regimes}

Here we discuss a method of predicting transition between the various flow regimes: no oscillations, shear-layer mode and wake mode. Our goal is not a quantitative prediction for the amplitude of oscillation, but rather an approximate scaling law to determine the parametric dependence of the transitions between the different regimes.

Our criteria for predicting transition to wake mode are based on the following observations: as certain parameters (e.g. $L / \theta_{0}$ ) are increased, the Kelvin-Helmholtz disturbances in the shear layer grow to larger amplitude. The larger-amplitude disturbances induce a larger recirculating flow within the cavity, possibly generating a region of absolute instability in the shear layer, and ultimately inducing large-scale vortex shedding from the leading edge. The key ideas here are that larger-amplitude disturbances lead to larger backflow in the cavity, which ultimately leads to wake mode.

The onset of fluctuations (i.e. the transition from steady flow to shear-layer mode) demonstrates a similar dependence on the parameters of the problem, since the amplification by the shear layer must exceed a certain threshold for self-sustained oscillations to occur (Woolley \& Karamcheti 1974). At low Mach number, Sarohia (1975) determined that there is a minimum cavity length, relative to the (laminar) incoming boundary-layer thickness, for which cavity oscillations may occur. For sufficiently large $D / \theta_{0}\left(D / \theta_{0}>\sim 15\right)$ he found oscillations only when

$$
\sqrt{R e_{\theta}} L / \theta_{0}>\sim 800 \text {, }
$$

which also shows that there is a minimum speed, a maximum viscosity, and a maximum boundary-layer thickness, holding other parameters constant, beyond which there are no oscillations. Gharib \& Roshko (1987) also measured the onset of fluctuations at $\sqrt{\operatorname{Re}_{\theta}} L / \theta_{0} \approx 780$. As $D / \theta_{0}$ was decreased below about 15 , Sarohia (1975) found a rapid increase in the minimum length for oscillations to occur. The 

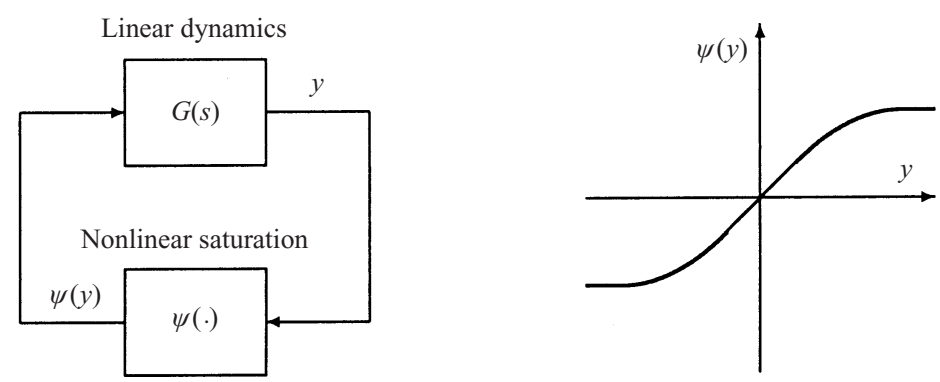

FIGURE 21. Simple model of nonlinearities.

data for turbulent fluctuations and higher Mach numbers are not so complete, but the general trend is that the minimum length increases with turbulence (Krishnamurty 1956; Sarohia 1975), and decreases with increasing Mach number (Krishnamurty 1956).

Thus, if the overall amplification of disturbances can be predicted, simple criteria for both the onset of fluctuations and the transition to wake mode would be that the amplification exceeds a certain value. In order to test this hypothesis, we estimate the amplification based on ideas about the shear-layer instability, and the efficiency of the acoustic radiation from the trailing edge. Although some of the approximations made are crude, our goal is to establish a rough parametric dependence of transitions between the flow regimes, rather than a detailed prediction for the overall amplitude.

\subsection{The role of nonlinearities}

All our analysis here will be linear. One might object at the outset that linear amplification rates say nothing about the final amplitude of a limit cycle-in fact, once the flow has settled into a limit cycle, the loop gain (total amplification once around the feedback loop) must be unity, regardless of the amplitude of the limit cycle (otherwise, oscillations would grow or decay). One must, in fact, take nonlinearities into account to predict amplitudes.

However, under simple yet reasonable assumptions about the nonlinearities present, the linear part of the loop gain defined above can predict both the onset of oscillations and the final amplitude of a limit cycle. Assume that the system may be approximated (crudely) by linear dynamics coupled with a frequency-independent nonlinear saturation, as shown in figure 21. Here, $G(s)$ is the transfer function of the linear elements in the model (for our purposes, this will be the shear-layer amplification and scattering at the downstream corner), and $\psi(\cdot)$ is a saturation nonlinearity, an odd function with $\psi(y) / y$ positive and decreasing for all $y>0$, and with $\psi(y) / y \rightarrow 0$ as $y \rightarrow \infty$ (for instance, $\psi(y)=\tanh (y)$ ). This form of the model lends itself to describing function analysis (e.g. Khalil 1996), a simple tool for approximating both frequencies and amplitudes of limit cycles in nonlinear systems.

If $y$ is periodic, of the form $y=A \sin \omega t$, then $\psi(y(t))$ will also be periodic, with the same frequency, and may be written as a Fourier series

$$
\psi(y(t))=\sum_{k=1}^{\infty} c_{k}(A) \sin k \omega t,
$$

where we have used that $\psi$ is odd. In the simplest form of describing function analysis, one neglects higher harmonics (assuming, for instance, that they will be attenuated 


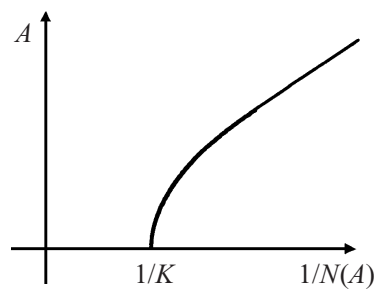

FIGURE 22. Describing function $N(A)$ for a saturation nonlinearity $\psi(\cdot)$. Here $K=\psi^{\prime}(0)$, the gain of $\psi(\cdot)$ for small amplitudes.

by $G(s)$ ), and considers only $k=1$, defining the describing function

$$
N(A)=\frac{c_{1}(A)}{A}=\frac{2}{\pi A} \int_{0}^{\pi} \psi(A \sin t) \sin t \mathrm{~d} t .
$$

Sinusoidal solutions $y=A \sin \omega t$ of the feedback loop must then satisfy $y=G(\mathrm{i} \omega) \psi(y)$ $\approx G(\mathrm{i} \omega) N(A) y$, which gives the harmonic balance equation

$$
G(\mathrm{i} \omega) N(A)=1 .
$$

For odd nonlinearities, $N(A)$ is real, and so taking the phase of this equation yields $\angle G(\mathrm{i} \omega)=0$, the same criterion used in Rossiter's model to determine possible frequencies of oscillation. Furthermore, taking the magnitude of (5.3) gives $|G(\mathrm{i} \omega)|=$ $1 / N(A)$, which determines the amplitude $A$ of the limit cycle. For a saturation nonlinearity as defined above, one can show that $N(A)$ is decreasing for $A>0$, $N(A) \rightarrow 0$ as $A \rightarrow \infty$, and $N(A) \rightarrow K$ as $A \rightarrow 0$, where $K=\psi^{\prime}(0)$ is the gain of $\psi(\cdot)$ for small amplitudes. The form of the describing function $N(A)$ is depicted in figure 22. From this, a simple stability argument demonstrates that for $|G(\mathrm{i} \omega)|<1 / K$, the frequency $\omega$ is stable (disturbances at that frequency will decay exponentially); if $|G(\mathrm{i} \omega)| \geqslant 1 / K$, finite-amplitude oscillations occur at that frequency (a stable limit cycle), and the amplitude $A$ of the limit cycle increases as $|G(\mathrm{i} \omega)|$ increases. This is clearly seen from figure 22 , recalling that $|G(\mathrm{i} \omega)|=1 / N(A)$ for a limit cycle of amplitude $A$ and frequency $\omega$.

Without knowing details of the nonlinearity and exact values for $G(\mathrm{i} \omega)$, we cannot predict the exact value of the amplitude. But if we obtain scaling laws for $G(\mathrm{i} \omega)$, the amplitude will follow (at least qualitatively) the same scaling laws: for $|G(\mathrm{i} \omega)|$ below a certain threshold, we expect no oscillations to occur, and for $|G(\mathrm{i} \omega)|$ above this threshold, we expect a stable limit cycle whose amplitude increases with $|G(\mathrm{i} \omega)|$.

The precise way in which nonlinearities enter the physics is still not clearly understood. One scenario, most likely to occur for short cavities (small $L / \theta_{0}$ ), is that employed by Cain et al. (1996), where the saturation occurs because of spreading of the shear layer: the spreading of the mean flow is caused by Reynolds stresses that are proportional to the square of the amplitude of the oscillations, plus any background turbulence or other nonlinear interactions between the modes, as described by Morris, Giridharan \& Lilley (1990). Linear growth rates of the Kelvin-Helmholtz instability waves decrease as the shear layer spreads, and eventually become negative for very thick shear layers, thus providing a saturation mechanism. For this mechanism, then, the shear-layer disturbances are described well by linear methods, given the correct shear-layer thickness. Our data in $\S 3.4$ are consistent with this, as shown by the good agreement of the data with linear predictions (see figure 13). Though the amplification 
predicted by linear theory does not agree well (figure 15), this is more likely because of viscous effects, rather than nonlinear effects, because there is disagreement even for small $x / \theta_{0}$.

The saturation mechanism more commonly considered is that once the oscillations grow large, the linearization is no longer valid, and the resulting nonlinear effects limit the growth. This effect is most likely for longer shear layers (large $L / \theta_{0}$ ), as in the experiments of Cattafesta et al. (1997), which had a very thin upstream boundary layer $\left(L / \theta_{0}=328\right)$. Knisely \& Rockwell (1982) also attributed their saturation to this mechanism, although it is also plausible that the saturation might be explained by mean flow spreading, as the linear stability calculation they compared to had a constant-thickness shear profile.

Both of these forms of nonlinearities fit nicely into the describing function framework described above: if a shear-layer disturbance at the leading edge is given by $f(0, t)=A \exp (\mathrm{i} \omega t)+$ c.c., then at a distance $x$ downstream, the amplified disturbance is approximately described by

$$
f(x, t)=N_{x}(A) A \exp (\mathrm{i} \omega t) G_{x}(\mathrm{i} \omega)+\text { c.c., }
$$

where $G_{x}(\mathrm{i} \omega)$ is the linear gain and phase at location $x\left(G_{x}(\mathrm{i} \omega)=\exp (\mathrm{i} \alpha(\omega) x)\right.$ for instance), and $N_{x}(A)$ is a nonlinear saturation, equal to unity for small $A$, and decreasing for large $A$, and also depending on the position $x$. Thus, $N_{x}(A)$ in (5.4) plays the same role as the describing function above, and similar conclusions about the stability and amplitude of the limit cycle follow.

\subsection{Shear layer}

The shear-layer amplification is a complicated function of $M, L / \theta_{0}, D / \theta_{0}$ and $\operatorname{Re}_{\theta}$. To proceed, we perform inviscid linear stability calculations similar to those described in $\S 3.4$ for each set of computational parameters in table 1 , and for each of the first three Rossiter frequencies (for which we use equation (3.1)). We assume a tanh velocity profile with a linear spreading rate $\mathrm{d} \delta_{\omega} / \mathrm{d} x=0.1$. Based on the discussion above and in $\S 3.3$, it is clear that the true spreading rate is dependent on growth of the disturbances in the layer, which, in turn, is dependent on all of $L / \theta_{0}, D / \theta_{0}, M$ and $R e_{\theta}$. However, we performed calculations using different values of the spreading rate, and found the amplitude variation to be relatively weak compared to the other factors.

\subsection{Scattering}

In order to assess the efficiency of the sound generation at the edge as a function of the Mach number, we use the simple model proposed by Tam \& Block (1978), where the process is idealized as an oscillating compact mass source (monopole). We determine the strength $Q$ of the monopole source by setting $Q$ equal to the mass flow rate out of the cavity. Tam points out that 'the unsteady mass addition and removal at the trailing edge of the cavity as the cause of the acoustic disturbance ... essentially suggests a dipole source at the trailing edge,' since by this mechanism a compression wave outside the cavity (mass addition) would correspond to a rarefaction wave inside the cavity (mass removal). However, as Tam points out, experimental, and now computational, observations indicate that the pressure waves inside and outside the cavity are in fact in phase, which corresponds to a monopole source at the trailing edge. Thus, we model the acoustic source as a monopole at the trailing edge, whose strength is determined by the mass flow rate out of the cavity. 
If density fluctuations are small, the mass flow rate per unit volume is given by

$$
Q=\frac{\dot{m}}{V} \approx \frac{\rho_{\infty}}{V} \int_{0}^{L} v(x, 0, t) \mathrm{d} x,
$$

where $v$ is the vertical velocity, and $V$ is the volume of the source, at this time unknown. Assuming that the velocity perturbations are sinusoidal, with an exponentially growing envelope, the integral above will be dominated by the portion closest to the trailing edge, and thus scales as $\lambda|v(L)|$, where $|v(L)|$ denotes the amplitude of the velocity perturbation near the trailing edge, and $\lambda$ is the wavelength of the instability wave. Expressing the wavelength as $\lambda=2 \pi c_{p} / \omega$, where $c_{p}$ is the phase speed of the instability wave, the source strength scales as

$$
\dot{Q}=\mathrm{i} \omega Q \propto \mathrm{i} \omega \frac{\rho_{0}}{V} \frac{c_{p}|v(L)|}{\omega} \propto \frac{\rho_{0} c_{p}|v(L)|}{V} .
$$

This expression may also be arrived at on dimensional grounds alone, if it is assumed that the monopole strength is proportional to the vertical velocity (or shear-layer displacement) at the trailing edge.

Density fluctuations then satisfy a Helmholtz equation (e.g. Crighton 1975), and far from the source are given by

$$
\frac{\rho}{\rho_{\infty}} \propto \frac{c_{p}|v(L)|}{a_{\infty}^{2}} \frac{\mathrm{e}^{\mathrm{i} k r}}{\sqrt{k r}},
$$

where $r$ is the distance from the source, and $k=\omega / a_{\infty}$ is the wavenumber. Note that there are two asymptotic arguments leading to equation (5.7). The first is that the retarded time is negligible over the source region (compact source), and the second is that we are at large distance, on the scale of the wavelength, from the source. The second approximation is not valid at very low Mach numbers, where the cavity length is small compared to the wavelength of the acoustic waves. For the present range of $M$, the asymptotic properties of the Green's function for the Helmholtz equation (i.e. the zeroth-order Hankel function of the first kind) can be used to show that there is negligible error in the second assumption.

At the cavity leading edge, $r=L$, we then have the following scaling law (assuming $c_{p} / U=$ const, as in the Rossiter model):

$$
\frac{|\rho(0)|}{\rho_{\infty}} \propto \frac{|v(L)|}{U} M^{3 / 2} S t^{-1 / 2},
$$

where $|\rho(0)|$ denotes the magnitude of density fluctuations at the leading edge, $x=0$.

\subsection{Loop gain}

We now have enough information to determine the scaling of the loop gain for the cavity: that is, the amplification of a disturbance as it is amplified by the shear layer and converted into a monopole acoustic source. (We do not include receptivity effects in this crude model.) Denoting the shear-layer amplification by $A\left(S t, M, L / \theta_{0}, D / \theta_{0}\right)$, the loop gain scales as

$$
\beta_{n} \propto A\left(S t_{n}, M, L / \theta_{0}, D / \theta_{0}\right) M^{3 / 2} S t_{n}^{-1 / 2},
$$

where $S t_{n}$ denotes the Strouhal number of Rossiter mode $n$, given by Rossiter's formula (3.1). The total amplification is then $\beta=\max _{n} \beta_{n}$, and we take $\beta$ as our criterion for predicting the onset of oscillations, and eventually wake mode. Note, 


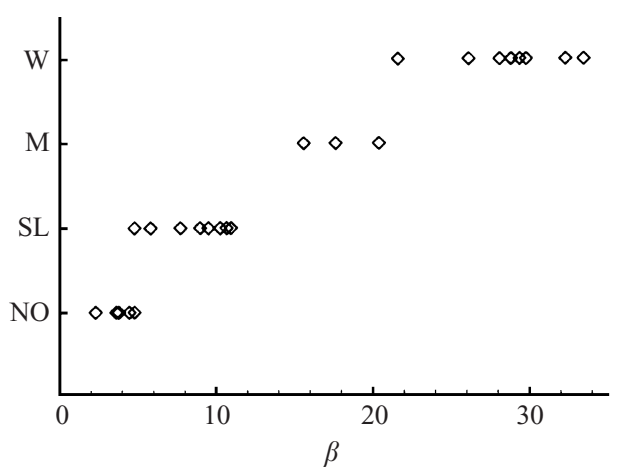

FIGURE 23. Correlation of loop gain $\beta$ with mode of cavity oscillations. All runs from table 1 with $56<R e_{\theta}<70$ are shown. As in table 1 , NO corresponds to no oscillations, SL denotes shear-layer mode, $\mathrm{M}$ denotes mixed mode, and $\mathrm{W}$ wake mode.
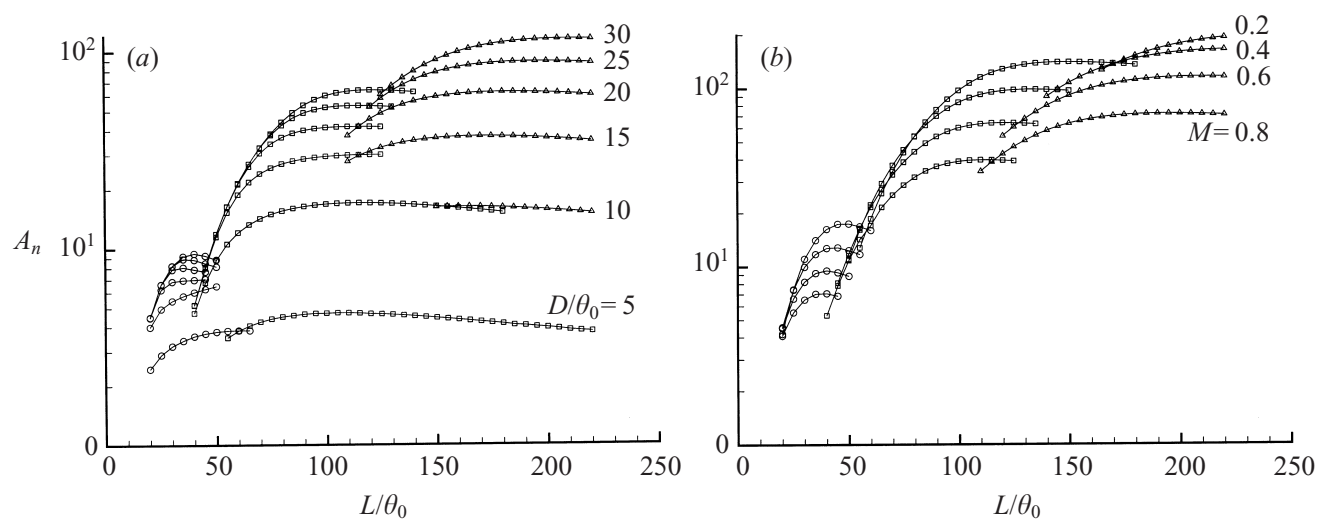

FiguRE 24. The shear-layer amplification, $A_{n}$, versus $L / \theta_{0} ;(a)$ for different $D / \theta_{0}$, with $M=0.6$; (b) for different $M$, with $D / \theta_{0}=30$. The dominant mode is shown at each $L / \theta_{0}$, with $n=1(\bigcirc)$, $n=2(\square)$, and $n=3(\triangle)$.

however, that the dependence on Reynolds number is not included in (5.9), as our shear-layer calculations were inviscid. Variation with Reynolds number is considered separately below.

Figure 23 shows how $\beta$ correlates with the mode of oscillation. Because Reynolds number effects are not included, we compare all runs in table 1 that fall in a limited Reynolds number range, $56<R e_{\theta}<70$. For each run, we compute the amplification $\beta_{n}$ for the first three Rossiter modes $(n=1,2,3)$, and $\beta$ for each run is then the maximum of the three. Figure 23 shows $\beta$ vs. the observed mode of cavity oscillation, and we find that for this Reynolds number range, $\beta<4.8$ corresponds to no oscillations, $4.8<\beta<15$ corresponds to shear-layer mode, $15<\beta<21$ to mixed mode, and $\beta>21$ to pure wake mode.

\subsection{Parametric dependence}

We now discuss some results related to the specific parametric dependence of the transitions. In figure 24(a), we have plotted the total amplification from the shear layer alone (corresponding to $A_{n}$ in equation (5.9)) for the first three Rossiter modes, as a function of $L / \theta_{0}$ and $D / \theta_{0}$, at $M=0.6$. Since, for fixed Mach number, the shear layer amplification is the predominant component of the loop gain $\beta$, the figure also 
indicates trends in $\beta$ as the cavity length is varied. The amplification shows strong dependence on $D / \theta_{0}$, especially for $D / \theta_{0}<\sim 20$. This explains very well the trend seen (for low Mach number) by Sarohia (1975), who found the minimum cavity length for oscillations increased dramatically for small $D / \theta_{0}$. The plot also shows why the higher Rossiter modes tend to dominate for longer cavities, as has been observed in experiments.

It is interesting to note that the predictions show that multiple modes are likely to be present at roughly equal amplitudes for cavities of certain length, while, for other lengths, one mode is clearly dominant. For example, for $L / \theta_{0}=125$ and $D / \theta_{0}=30$, modes 2 and 3 should be of roughly equivalent strength (ignoring nonlinear interactions and frequency dependence of scattering/receptivity).

Figure 24(a) also shows similar effects for the transition to wake mode as a function of $D / \theta_{0}$, but given that the amplitude threshold is higher, wake mode should not occur in cavities with $D / \theta_{0}<\sim 20$.

In figure $24(b)$ the total amplification in the shear layer for the first three Rossiter modes is plotted as a function of $L / \theta_{0}$ and $M$, for $D / \theta_{0}=30$. The amplification rates are highest for the lowest Mach numbers, which is consistent with many previous analyses of the Kelvin-Helmholtz instability in compressible shear layers. This decreasing amplitude must be balanced by the increase in radiation efficiency at the edge as $M$ is increased. As noted above, it is observed in experiments (Krishnamurty 1956) that the minimum cavity length for the onset of fluctuations decreases with $M$. This is indeed what the model predicts, as evidenced by the good agreement with our data in figure 23.

We note that the computational data also show a tendency for transition to wake mode with increasing Reynolds number. The R4 series of runs, for example, show a change from shear-layer to wake mode for $L / \theta_{0} \approx 75$ as $R e_{\theta}$ varies from 40 to 80 . The model of the transition process discussed above is based on an inviscid stability analysis, and is therefore unable to predict any Reynolds number effects. However, for the range of low Reynolds numbers, substantial reduction in the amplitude gain in the shear layer may be expected (e.g. Michalke 1984), which is consistent with figure 15, and with the observed trend in transition to wake mode.

It has been noted above that the boundary layer periodically separates and reattaches upstream of the cavity during wake mode oscillations. This is true for all runs where we have observed the transition to wake mode, and it is likewise apparently the case in the experiments of Gharib \& Roshko (1987). Separation was not observed in any of the runs with shear-layer mode oscillations. By carefully examining the evolution of the flow over time, it is also observed that this periodic separation of the upstream boundary layer precedes the transition of the flow to its nearly periodic wake mode state. Thus it appears that this separation is intimately connected with the transition to wake mode, though we have not been able to establish any causal relationship $\dagger$

\section{Concluding remarks}

We have used numerical simulations to explore the operating regimes of the laminar flow past a two-dimensional rectangular cavity. For short cavities (relative to the upstream boundary-layer thickness), and for low Mach numbers and Reynolds

$\dagger$ In a preliminary report on this work (Colonius et al. 1999), we stated that the unsteady separation appeared to be the cause of wake mode; in retrospect, this statement was too strong. 
numbers, the flow is steady. As these parameters are increased, the flow transitions into a shear-layer mode, where self-sustained oscillations occur. This is the regime usually observed in experiments, and the acoustic fields predicted by the simulation agree well with schlieren photographs from the experiments by Krishnamurty (1956). The growth of disturbances in the shear layer is predicted well by a locally parallel linear stability calculation, where the thickness of the shear layer is measured from the simulations.

For longer cavities, and larger Mach and Reynolds numbers, the flow transitions into a wake mode. Similar flow features have been observed in axisymmetric cavity experiments by Gharib \& Roshko (1987), and in experiments in a pipe with closed side branches (Kriesels et al. 1995). The frequency of oscillations in wake mode is independent of Mach number, indicating a purely hydrodynamic (non-acoustic) instability. In wake mode, a significant backflow is present inside the cavity, and we hypothesize that this backflow leads to an absolute instability, which may provide the feedback mechanism leading to wake mode. We have used a simple linear model to predict the scaling laws governing transition between the flow regimes, and the model agrees well with data from our simulations.

To our knowledge wake mode oscillations have not been observed in experiments on shallow cavities in a similar range of $L / D$ to that considered here (although very recent three-dimensional PIV measurements (A. Krothapalli 2001, personal communication) in an $L / D=5$ cavity show on average a significant recirculating flow, with backflow velocities reaching about $15 \%$ of the free-stream velocity). The experiments of Krishnamurty (1956) are closest to the parameters of the present case (the Reynolds number being typically 5 times larger than in the present flow), but even for very shallow (large $L / D$ ) cases his schlieren photographs do not show evidence of a transition to wake mode. On the other hand, his longest cavities had very thick upstream shear layers (the length was held constant while decreasing the depth, with constant upstream conditions). And as shown in the previous section, wake mode is not indicated for $D / \theta_{0}<\sim 15$.

According to the model proposed in the previous section, wake mode oscillations should occur when the amplitude of oscillation increases beyond a certain threshold, such that the recirculating flow in the cavity is sufficiently strong to cause a shift to absolute instability of the shear layer. Thus, any effect that tends to decrease the amplitude of the fluctuations could inhibit the transition to wake mode (or at least modify the location in parameter space where the transition occurs). It is known that when the upstream boundary layer is turbulent, the radiated acoustic field is less intense (Krishnamurty 1956), and the minimum length for oscillations to occur is increased (Sarohia 1975). Thus boundary-layer turbulence should inhibit the transition to wake mode. Three-dimensional effects beyond turbulence in the boundary layer, such as cavity sidewalls, may sufficiently change the character of the recirculating flow in the cavity and also inhibit the transition to wake mode. Recent work by Barkley, Gomes \& Henderson (1999) shows that the first global instability of a steady two-dimensional flow over a backward-facing step is a steady three-dimensional mode localized within the separation bubble. The spanwise wavelength of the mode is about 7 step heights, and they suggest that the mode may be caused by centrifugal instability within the separation bubble. The critical Reynolds number found in their calculations was 748, which is smaller than the Reynolds number of the present simulations (based in our case on the cavity depth) by a factor of about 2 . It is plausible that similar instabilities may be present within the recirculating flow in the shallow cavity, and that such instabilities could significantly modify the transition to wake mode. 
It is not possible to resolve these issues without recourse to more detailed threedimensional simulations and experiments. It is our hope that the model proposed here for the transition may help guide future efforts to fully characterize the transition to wake mode in more general turbulent and three-dimensional flows.

This research was supported by AFOSR under grant F49620-98-1-0095 with technical monitor Dr Thomas Beutner. Supercomputer time was provided by the Department of Defense High Performance Computing centers, as well as the National Science Foundation. The first author acknowledges the support of a National Science Foundation Graduate Fellowship. We wish to thank Drs Anatol Roshko, Alan Cain, David Williams, Drazen Fabris, and Edward Kerschen for many helpful discussions on cavity oscillations.

\section{REFERENCES}

Ahuja, K. K. \& Mendoza, J. 1995 Effects of cavity dimensions, boundary layer, and temperature on cavity noise with emphasis on benchmark data to validate computational aeroacoustic codes. Final Report Contract NAS1-19061, Task 13. NASA Contractor Report.

Barkley, D., Gomes, M. G. M. \& Henderson, R. 1999 Three-dimensional instability in flow over a backward-facing step. Submitted to J. Fluid Mech.

Blake, W. K. \& Powell, A. 1986 The development of contemporary views of flow-tone generation. In Recent Advances in Aeroacoustics, pp. 247-345. Springer.

Brown, G. L. \& Roshko, A. 1974 On density effects and large structure in turbulent mixing layers. J. Fluid Mech. 64, 775-816.

Cain, A. B., Bower, W. W., McCotter, F. \& Romer, W. W. 1996 Modeling and prediction of weapons bay acoustic amplitude and frequency. Tech. Rep. VEDA Inc.

Cattafesta, III, L. N., Garg, S., Choudhari, M. \& Li, F. 1997 Active control of flow-induced cavity resonance. AIAA Paper 97-1804.

Cattafesta, III, L. N., Garg, S., Kegerise, M. S. \& Jones, G. S. 1998 Experiments on compressible flow-induced cavity oscillations. AIAA Paper 98-2912.

Colonius, T. 2001 An overview of simulation, modeling, and active control of flow/acoustic resonance in open cavities. AIAA Paper 2001-0076.

Colonius, T., Basu, A. J. \& Rowley, C. W. 1999 Numerical investigation of the flow past a cavity. AIAA Paper 99-1912.

Colonius, T., Lele, S. K. \& Moin, P. 1993 Boundary conditions for direct computation of aerodynamic sound. AIAA J. 31, 1574-1582.

Colonius, T., Lele, S. K. \& Moin, P. 1997 Sound generation in a mixing layer. J. Fluid Mech. 330, 375-409.

Crighton, D. G. 1975 Basic principles of aerodynamic noise generation. Prog. Aerosp. Sci. 16, $31-96$.

Crighton, D. G. 1992 The jet edge-tone feedback cycle; linear theory for the operating stages. J. Fluid Mech. 234, 361-391.

Crighton, D. G. \& Gaster, M. 1976 Stability of slowly diverging jet flow. J. Fluid Mech. 77, 397-413.

Fox, J. 1965 Flow regimes in transverse rectangular cavities. In Proc. 1965 Heat Transfer and Fluid Mechanics Institute. Stanford University Press.

Fox, J. 1968 A criterion for the transition between flow regimes in turbulent cavity flow. AIAA J. 4, $364-365$.

Freund, J. B. 1997 Proposed inflow/outflow boundary condition for direct computation of aerodynamic sound. AIAA J. 35, 740-742.

Fuglsang, D. F. \& CAIN, A. B. 1992 Evaluation of shear layer cavity resonance mechanisms by numerical simulation. AIAA Paper 92-0555.

Gharib, M. 1987 Response of the cavity shear layer oscillations to external forcing. AIAA J. 25, $43-47$. 
Gharib, M. \& Roshko, A. 1987 The effect of flow oscillations on cavity drag. J. Fluid Mech. 177, $501-530$.

Heller, H. H. \& Bliss, D. B. 1975 The physical mechanism of flow induced pressure fluctuations in cavities and concepts for their suppression. AIAA Paper 75-491.

Howe, M. S. 1997 Edge, cavity and aperture tones at very low mach numbers. J. Fluid Mech. 330, 61-84.

Huerre, P. \& Monkewitz, P. A. 1985 Absolute and convective instabilities in free shear layers. J. Fluid Mech. 159, 151-168.

Huerre, P. \& Monkewitz, P. A. 1990 Local and global instabilities in spatially developing flows. Annu. Rev. Fluid Mech. 22, 473-537.

Khalil, H. K. 1996 Nonlinear Systems, 2nd edn. Prentice-Hall.

KNisely, C. \& Rockwell, D. 1982 Self-sustained low-frequency components in an impinging shear layer. J. Fluid Mech. 116, 157-186.

Kriesels, P. C., Peters, M. C. A. M., Hirschberg, A., Wijnands, P. J., Iafrati, A., Riccardi, G., Piva, R. \& Bruggeman, J. C. 1995 High amplitude vortex-induced pulsations in a gas transport system. J. Sound Vib. 184, 343-368.

KrishnamurTy, K. 1956 Sound radiation from surface cutouts in high speed flow. PhD thesis, California Institute of Technology.

Lamp, A. M. \& ChOKANI, N. 1997 Computation of cavity flows with suppression using jet blowing. J. Aircraft 34, 545-551.

LeLE, S. K. 1992 Compact finite difference schemes with spectral-like resolution. J. Comput. Phys. $103,16-42$.

Michalke, A. 1984 Survey on jet instability theory. Prog. Aerospace Sci. 21, 159-199.

Monkewitz, P. A. \& NGUYEN, L. N. 1987 Absolute instability in the near-wake of two-dimensional bluff bodies. J. Fluids Struct. 1, 165-184.

Morris, P. J., Giridharan, M. G. \& Lilley, G. M. 1990 On the turbulent mixing of compressible free shear layers. Proc. R. Soc. Lond. A 431, 219-243.

Poinsot, T. \& LeLE, S. K. 1992 Boundary conditions for direct simulation of compressible viscous flows. J. Comput. Phys. 101, 104-129.

Powell, A. 1953 On edge tones and associated phenomena. Acustica 3, 233-243.

Powell, A. 1961 On the edgetone. J. Acoust. Soc. Am. 33, 395-409.

RockWELl, D. \& NAUDASCHER, E. 1978 Review-self-sustaining oscillations of flow past cavities. Trans. ASME: J. Fluids Engng 100, 152-165.

Rockwell, D. \& Schachenmann, A. 1982 Self-generation of organized waves in an impinging turbulent jet at low Mach number. J. Fluid Mech. 117, 425-441.

Roshko, A. 1955 Some measurements of flow in a rectangular cutout. NACA TN 3488.

Rossiter, J. E. 1964 Wind-tunnel experiments on the flow over rectangular cavities at subsonic and transonic speeds. Aero. Res. Counc. R\&M, No. 3438.

Rowley, C. W. \& Colonius, T. 2000 Discretely nonreflecting boundary conditions for linear hyperbolic systems. J. Comput. Phys. 157, 500-538.

SAROHIA, V. 1975 Experimental and analytical investigation of oscillations in flows over cavities. $\mathrm{PhD}$ thesis, California Institute of Technology.

Sarohia, V. \& Massier, P. F. 1977 Control of cavity noise. J. Aircraft 14, 833-837.

ShIEH, C. M. \& Morris, P. J. 1999 Parallel numerical simulation of subsonic cavity noise. AIAA Paper 99-1891.

Slimon, S., Davis, D. \& Wagner, C. 1998 Far field aeroacoustic computation of unsteady cavity flows. AIAA Paper 98-0285.

TAM, C. K. W. \& BLock, P. J. W. 1978 On the tones and pressure oscillations induced by flow over rectangular cavities. J. Fluid Mech. 89, 373-399.

Thompson, K. W. 1987 Time dependent boundary conditions for hyperbolic systems. J. Comput. Phys. 68, 1-24.

Woolley, J. P. \& Karamcheti, K. 1974 Role of jet stability in edgetone generation. AIAA J. 12, $1456-1458$.

Zhang, X., Rona, A. \& Edwards, J. A. 1998 The effect of trailing edge geometry on cavity flow oscillation driven by a supersonic shear layer. Aeronaut. J. 102, 129-136. 